\title{
Differential geometry of gauge theories: an introduction
}

\author{
Jordan François ${ }^{a, *}$ \\ ${ }^{a}$ Service de Physique de l'Univers, Champs et Gravitation, Université de Mons - UMONS, \\ 20 Place du Parc, B-7000 Mons, Belgium \\ E-mail: jordan.francois@umons.ac.be
}

We propose an introduction to the differential geometry of connections on fiber bundles underlying the physics of (classical) gauge theories. Reminding first how Ehresmann connections are behind Yang-Mills-Utiyama theories, we will then be prepared to appreciate why Cartan connections provide a compelling geometric framework for gauge theories of gravity. A recipe of sort is then given, showing how these geometric data provide a kinematics that constrain and channel the construction of gauge theories, which specify the dynamics of the geometry. We end with a brief presentation of the "dressing field method", a systematic tool to achieve gauge symmetry reduction that has a wide range of applications in gauge theory.

XVI Modave Summer School in Mathematical Physics - Modave 2020

9-11 September 2020

Brussels, Belgium

${ }^{*}$ Speaker 


\section{Contents}

1 Introduction \& motivation 2

2 Some basics 4

3 Manifolds: the 4 levels $\quad 6$

$\begin{array}{ll}3.1 & \text { Topological manifolds } \\ 3.2 & \text { Difleral }\end{array}$

$\begin{array}{lll}3.2 & \text { Differential manifolds } & 7\end{array}$

3.3 Manifolds with connections 9

$\begin{array}{ll}3.4 \text { Metric manifolds } & 10\end{array}$

4 Principal bundles and connections $\quad 12$

$\begin{array}{lll}4.1 & \text { Principal bundles and their smooth structure } & 12\end{array}$

$\begin{array}{ll}4.2 \text { Ehresmann connections } & 15\end{array}$

$\begin{array}{lll}4.3 \text { Cartan connections } & 16\end{array}$

$\begin{array}{ll}4.4 \text { Gauge theories: a recipe } & 18\end{array}$

4.4.1 Yang-Mills-Utiyama gauge theories 18

$\begin{array}{lll}\text { 4.4.2 Gravitational gauge theories } & 20\end{array}$

5 The dressing field method 22

5.1 Reduction of gauge symmetries 22

$\begin{array}{lll}5.2 & \text { Residual gauge transformations } & 23\end{array}$

6 Conclusion $\quad 24$

\section{Introduction \& motivation}

The advent of General Relativity (GR) was a pivotal moment for both the history of physics and mathematics. On the mathematical side, it sparked an era of renewed interest in differential geometry whose earliest fruit is the theory of parallel transport and connection by Levi-Civita and Schouten. Weyl's 1918-19 attempt at a generalisation of this theory, was followed in the 20's by other studies of variants of connections on manifolds, culminating with Cartan's espace généralisés equipped with Cartan connections [1]. In the 30's and 40's, abstract spaces - fibered spaces - were defined [2,3] and studied [4,5], when Ehresmann finally gave his general definition of a connection on such spaces [6]. In the 50's the differential geometry of connections on fiber bundles flourished [7], with major reference texts in the 60's [8,9] signaling the domain had reached maturity. 
On the physical side, GR inspired Weyl's 1918 theory [10,11] which was the first attempt at a unified theory of gravitation and electromagnetism, relating in particular charge conservation to a new geometric symmetry in addition to coordinate change invariance: local "gauge" or scale invariance, which is what we would call today conformal - or Weyl - rescalings. The idea resurfaced after the advent of Quantum Mechanics in 1925-26, as a local phase "rescaling" of the wave function. In 1929, Weyl [12] gave the first modern articulation of the gauge principle in electromagnetic (EM) theory coupled to a quantum electron: arguing that the theory's structure, minimal coupling and charge conservation come from a requiring its invariance under local phase transformations, $\mathcal{U}(1)$, which he kept calling gauge transformations. In 1954 Yang and Mills [13], R. Shaw [14], and R. Utiyama [15] all independently understood how to built a $\mathcal{S U}(2)$ gauge theory (Utiyama understood the procedure for any Lie group). But it was soon appreciated that gauge symmetries forces interaction mediating gauge fields to be massless. A fact a priori hard to reconcile with the very short range of the nuclear interactions, which suggested - following Yukawa's 1935 proposal [16] - that they were mediated by very massive bosons. Yet, in the late 60's and early 70's the gauge structure of the electro-weak [17] and strong interaction [18] was made clear thanks to the discovery of the spontaneous symmetry breaking (SSB) mechanism [19-21] and of asymptotic freedom (and confinement) of quarks $[22,23]$. By the mid to late 70's, the standard model (SM) of particle physics, a $\mathcal{U}(1) \times \mathcal{S U}(2) \times \mathcal{S U}(3)$ gauge field theory, was essentially achieved, and since then its predictions keep being confirmed to a high degree of accuracy.

The above sketch does not do justice to the glorious details of the histories of both subjects. So, for a historical account of the roots and development of gauge theory see [24]. For a broader history of the development of particle physics (up to 1984 and the discovery of the weak bosons) see the masterful book by Pais [25]. For an enlightening selection of papers retracing the development of the geometric conception of space and spacetime from Riemann to Einstein and Cartan, see [26]. For a historical contextualisation of the emergence of Ehresmann connections see [27].

The early 70's was also the moment when some started to realise that the theory of connections on fiber bundles was the mathematical underpinning of (classical) gauge theories [28-30]. After going their own separate ways for almost four decades, differential geometry and theoretical physics thus met again, and then began a golden age of cross-fertilisation of both fields. This synergy is still ongoing today, as differential geometric concepts and tools play an increasing important role.

Hence these short lecture notes, which aim to introduce the unfamiliar reader to the basics of the differential geometry of connections on manifolds and fiber bundles. We try to be relatively systematic, thus in section 2 we defined various relevant algebraic concepts, in section 3 we take care to distinguish the different structure levels on a manifold. In section 4, we describe principal bundles and define both Ehresmann and Cartan connections. The latter in particular are not widely known, yet Cartan geometry is, we argue, the best fit for classical gauge theories of gravity. We then consider how gauge theories are built from these geometric data. In the fifth and last section, we motivate and advertise a tool of gauge symmetry reduction known as the "dressing field method". For complements on the technical aspects presented in this lecture notes, a very readable reference is [31]. A classic introductory text covering a broad range of topics is [32]. More advanced reference mathematical texts on Cartan geometry are [33] and [34], and for its application to gravity one can consult e.g. [35, 36]. Since we have given a historical introduction to this basic technical review of gauge theories, we will conclude with more philosophical commentaries. 


\section{Some basics}

In the following, $\mathbb{K}$ denotes any underlying field, but is often meant to stand for either $\mathbb{R}$ or $\mathbb{C}$.

Definition 1. A graded vector space $\mathrm{V}$ decomposes as a direct sum $\mathrm{V}=\bigoplus_{k \in \mathbb{N}} \mathrm{V}^{k}$, where $k$ is the degree of elements in the vector subspaces $\mathrm{V}^{k}$. Any $v \in \mathrm{V}^{k}$ is said homogeneous of degree $|v|=k$. By convention $\mathrm{V}^{0}=\mathbb{K}$.

Definition 2. The dual of a $\mathbb{K}$-vector space $V$ is the space $\mathcal{L}(V, \mathbb{K})$ of linear maps $V \rightarrow \mathbb{K}$. It is itself a vector space, noted $V^{*}$. That is, $\omega \in V^{*}$ is $\omega: V \rightarrow \mathbb{K}, X \mapsto \omega(X)$, and s.t. $\omega(\lambda X)=\lambda \omega(X)$ for $\lambda \in \mathbb{K}$ and $\omega(X+Y)=\omega(X)+\omega(Y)$ for $X, Y \in V$. Elements of $V^{*}$ are called covectors. In the finite dimensional setting $\operatorname{dim} V=n=\operatorname{dim} V^{*}$.

Given a basis $\left\{e_{i}\right\}_{i \in[1 \cdots n]}$ of $V$ s.t. $X=X^{i} e_{i}$ for any $X \in V$, one has the dual basis $\left\{e^{i}\right\}$ of $V^{*}$ s.t. $\omega=\omega_{i} e^{i}$ for any $\omega \in V^{*}$ and s.t. $e^{i}\left(e_{j}\right)=\delta_{j}^{i}$. The basis allows to write $\omega(X)=\omega_{i} X^{i}$.

Definition 3. An algebra, $A$, is a vector space $V$ endowed with a bilinear map $\cdot: V \times V \rightarrow V$ called the product, which can be non-commutative or even non-associative (and may also be required to satisfy further desiderata). An important class of examples is provided by Lie algebras, whose product is the Lie bracket $[]:, L \times L \rightarrow L,(X, Y) \mapsto[X, Y]$, which is antisymmetric (thus non-commutative) and satisfies the Jacobi identity: $[X,[Y, Z]]+[Y,[Z, X]]+[Z,[X, Y]] \equiv 0$ (thus is non-associative). An algebra can be turned into a Lie algebra by defining the bracket $[X, Y]:=X \cdot Y-Y \cdot X$.

For a graded algebra $A$ the product is $\cdot: \mathrm{V}^{a} \times \mathrm{V}^{b} \rightarrow \mathrm{V}^{a+b},(A, B) \mapsto A \cdot B$. As a subclass, we have graded Lie algebras whose Lie bracket satisfies $[A, B]=-(-)^{a b}[B, A]$ and the graded Jacobi identity: $(-)^{a c}[A,[B, C]]+(-)^{b a}[B,[C, A]]+(-)^{c b}[C,[A, B]] \equiv 0$. A graded algebra $\mathrm{A}$ is a graded Lie algebra with the bracket $[A, B]:=A \cdot B-(-)^{a b} B \cdot A$.

Definition 4. A derivation homogeneous of degree $|d|$ on a graded algebra $A$ is a linear map $d: \mathrm{A}^{k} \rightarrow \mathrm{A}^{k+|d|}$ satisfying a graded Leibniz identity: $d(A \cdot B)=d A \cdot B+(-)^{a|d|} A \cdot d B$. If $|d|$ is odd, $d$ is an antiderivation. The pair $(A, d)$ is a graded differential algebra.

The space of derivations of $A$ is noted $\operatorname{Der}(A)$, it is clearly a vector space under addition. It is also a (graded) Lie algebra with the (graded) bracket $\left[d, d^{\prime}\right]:=d \circ d^{\prime}-(-)^{|d|\left|d^{\prime}\right|} d^{\prime} \circ d$.

For any $A \in \mathrm{A},[A,] \in \operatorname{Der}(\mathrm{A})$ is an inner derivation. Inner derivations are noted $\operatorname{Inn}(\mathrm{A}) \subset \operatorname{Der}(\mathrm{A})$.

\section{Definition 5. The exterior, or Grassmann, algebra of $\boldsymbol{V}$ :}

$$
\overbrace{V \times \cdots \times V}^{k \text { times }}
$$

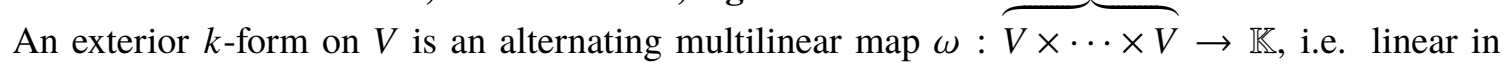
each argument and antisymmetric in the exchange of any two arguments. A covector is an exterior 1 -form. Note $\Lambda^{k}(V, \mathbb{K})$ the vector space of exterior $k$-forms on $V$. Clearly there cannot be $k$-forms with $k>n$. The graded vector space of all exterior forms on $V$ is then $\Lambda^{\bullet}(V, \mathbb{K})=\bigoplus_{k=0}^{n} \Lambda^{k}(V, \mathbb{K})$. The exterior, or wedge, product $\wedge: \Lambda^{a}(V, \mathbb{K}) \times \Lambda^{b}(V, \mathbb{K}) \rightarrow \Lambda^{a+b}(V, \mathbb{K}),(\alpha, \beta) \mapsto \alpha \wedge \beta$, defined as

$(\alpha \wedge \beta)\left(X_{1}, \cdots, X_{a}, X_{a+1}, \cdots X_{a+b}\right):=\frac{1}{a ! b !} \sum_{\sigma}|\sigma| \alpha\left(X_{\sigma(1)}, \cdots, X_{\sigma(a)}\right) \beta\left(X_{\sigma(a+1)}, \cdots, X_{\sigma(a+b)}\right)$

where $\sigma$ is a permutation and $|\sigma|= \pm$ its signature, satisfies $\alpha \wedge \beta=(-)^{a b} \beta \wedge \alpha$, making $\Lambda^{\bullet}(V, \mathbb{K})$ a graded commutative algebra known as the exterior, or Grassmann, algebra of $V$. 
$\boldsymbol{R} \boldsymbol{m} \boldsymbol{k}$ : Notice that in its definition, the wedge product uses the product of the field $\mathbb{K}$ in which the forms take values. So it can still be defined on exterior forms with values in an algebra $A$, $\Lambda^{\bullet}(V, A)=\Lambda^{\bullet}(V, \mathbb{K}) \otimes A$. If the product in $A$ is not commutative, the wedge product on $\Lambda^{\bullet}(V, A)$ is s.t. $\alpha \wedge \beta \neq(-)^{a b} \beta \wedge \alpha$. On exterior forms with values in a vector space $E, \Lambda^{\bullet}(V, E)=\Lambda^{\bullet}(V, \mathbb{K}) \otimes E$, the wedge product cannot be defined.

Given a basis of $V^{*}, \omega \in \Lambda^{k}(V, \mathbb{K})$ is $\omega=\frac{1}{k !} \omega_{i_{1} \cdots i_{k}} e^{i_{1}} \wedge \cdots \wedge e^{i_{k}}$, and $\omega_{i_{1} \cdots i_{k}}$ are the components of $\omega$ in the basis $\left\{e^{i}\right\}$ (forming a totally antisymmetric tensor), and $e^{i_{1}} \wedge \cdots \wedge e^{i_{k}}$ is the basis of $k$-forms. Notice there are $\left(\begin{array}{l}n \\ k\end{array}\right)$ elements in this basis, $\operatorname{so} \operatorname{dim} \Lambda^{k}(V, \mathbb{K})=\left(\begin{array}{l}n \\ k\end{array}\right)$ and

$$
\operatorname{dim} \Lambda^{\bullet}(V, \mathbb{K})=\sum_{k=0}^{n} \operatorname{dim} \Lambda^{k}(V, \mathbb{K})=\sum_{k=0}^{n}\left(\begin{array}{l}
n \\
k
\end{array}\right)=2^{n} .
$$

Definition 6. Metric: A non-degenerate metric on $V$ is a symmetric bilinear map $g: V \times V \rightarrow \mathbb{R}$, $(X, Y) \mapsto g(X, Y)$ of signature $(r, s)$ - or simply $s$, the number of negative eigenvalues.

Given a basis $\left\{e_{i}\right\}$ of $V$, the components of $g$ are given by $g_{i j}:=g\left(e_{i}, e_{j}\right)$. So that $g(X, Y)=g_{i j} X^{i} Y^{j}$. For $X \in V$, the covector/exterior 1-form $g(X):, V \rightarrow \mathbb{K} \in \mathcal{L}(V, \mathbb{K})=V^{*}$ is called the dual of $X$. In component this is $g(X,)=,g_{i j} X^{i} e^{j}=: X_{j} e^{j}$.

Definition 7. Hodge duality: Since $\left(\begin{array}{c}n \\ k\end{array}\right)=\left(\begin{array}{c}n \\ n-k\end{array}\right)$ we have $\operatorname{dim} \Lambda^{k}(V, \mathbb{K})=\operatorname{dim} \Lambda^{n-k}(V, \mathbb{K})$. So there is an isomorphism between the vector spaces of $k$-forms and $(n-k)$-forms. Given a metric $g$ on $V$ with signature $s$, an explicit realisation of this isomorphism is provided by the Hodge duality operator: $*: \Lambda^{k}(V, \mathbb{K}) \rightarrow \Lambda^{n-k}(V, \mathbb{K})$. It acts on the basis as,

$$
e^{i_{1}} \wedge \cdots \wedge e^{i_{k}} \mapsto *\left(e^{i_{1}} \wedge \cdots \wedge e^{i_{k}}\right):=\frac{|\operatorname{det}(g)|^{1 / 2}}{(n-k) !} \varepsilon_{j_{1} \cdots j_{n}} g^{i_{1} j_{1}} \cdots g^{i_{k} j_{k}} e^{j_{k+1}} \wedge \cdots \wedge e^{j_{n}} .
$$

So on $\omega \in \Lambda^{k}(V, \mathbb{K})$ we have,

$$
* \omega=\frac{1}{k !} \omega_{i_{1} \cdots i_{n}} *\left(e^{i_{1}} \wedge \cdots \wedge e^{i_{k}}\right)=\frac{1}{(n-k) !}(* \omega)_{i_{k+1} \cdots i_{n}} e^{i_{k+1}} \wedge \cdots \wedge e^{i_{n}},
$$

where $(* \omega)_{i_{k+1} \cdots i_{n}}$ is the "dual tensor" of $\omega_{i_{1} \cdots i_{n}}$. It is a useful exercise to write it explicitly. Another exercise is to prove that,

$$
\begin{aligned}
*^{2}=* \circ *: \Lambda^{k}(V, \mathbb{K}) & \rightarrow \Lambda^{k}(V, \mathbb{K}) \\
\omega & \mapsto * * \omega=(-)^{k(n-k)+s} \omega .
\end{aligned}
$$

Definition 8. Chain / cochain complexes, and cohomology:

A cochain complex $\left(C^{k}, f_{k}\right)$ is a sequence of abelian groups and morphisms $f$,

$$
\cdots \longrightarrow C^{k-1} \underset{g_{k}}{\stackrel{f_{k-1}}{\rightleftarrows}} C^{k} \underset{g_{k+1}}{\stackrel{f_{k}}{\rightleftarrows}} C^{k+1} \longrightarrow \cdots
$$

where $f_{k} \circ f_{k-1} \equiv 0$, or $f^{2} \equiv 0$. A chain complex $\left(C^{k}, g_{k}\right)$ is a sequence of abelian groups and morphisms $g$ s.t. $g_{k} \circ g_{k+1} \equiv 0$, or $g^{2} \equiv 0$. We focus on cochain complexes, but definitions mutatis mutandis apply to chain complexes. En element $c \in C^{k}$ is a $k$-cochain, and it is said:

- closed, and called a $k$-cocycle, if $f_{k}(c)=0$. One writes $c \in Z^{k}:=\operatorname{ker} f_{k} \subset C^{k}$.

- exact, and called a $k$-coboundary, if $c=f_{k-1}(b)$ for some $b \in C^{k-1}$. Write $c \in B^{k}:=\operatorname{Im} f_{k-1} \subset Z^{k}$.

The $k^{\text {th }}$-cohomology group is $H^{k}:=Z^{k} / B^{k}$, i.e. it is the group of closed but not exact $k$-cochains. The cohomology of the cochain complex is $H^{\bullet}=\bigoplus_{k} H^{k}$. 


\section{Definition 9. Short Exact Sequence (SES):}

An exact sequence is a cochain complex s.t. $\operatorname{Im} f_{k-1}=\operatorname{ker} f_{k}$ (it is said acyclic), i.e. its cohomology is trivial, $H^{\bullet} \equiv 0$. A short exact sequence (SES) is,

$$
0 \longrightarrow A \stackrel{\iota}{\longrightarrow} B \stackrel{\pi}{\longrightarrow} C \longrightarrow 0
$$

where $\iota$ is injective, $\pi$ is surjective, and $\pi \circ \iota=0$ so that $C \simeq B / \iota(A)$. The SES is said split if either - $\exists$ a section of $\pi, s: C \rightarrow B$ s.t. $\pi \circ s=\operatorname{id}_{C}$, or equivalently if

$-\exists$ a retraction of $\iota, r: B \rightarrow A$ s.t. $r \circ \iota=\mathrm{id}_{A}$.

Then one has $B \simeq \iota(A) \oplus s(C)$.

$$
0 \longrightarrow A \underset{r}{\longrightarrow} B \underset{s}{\longrightarrow} C \longrightarrow 0
$$

SES appear naturally in various area of mathematics. For example they are important in the definition of Lie algebras extensions and their theory. As a first example, derivations of a (graded) algebra $A$ give rise to the SES

$$
0 \longrightarrow \operatorname{Inn}(A) \stackrel{\iota}{\longrightarrow} \operatorname{Der}(A) \stackrel{\pi}{\longrightarrow} \operatorname{Der}(A) / \operatorname{Inn}(A) \longrightarrow 0 .
$$

Splitting of this sequence is at the heart of derivation-based non-commutative geometry, which is one way to build Yang-Mills-Higgs gauge theories [37]. As we will see, SES also appear in the geometry of fibered spaces.

\section{Manifolds: the 4 levels}

In this section we review basic material about (real) manifolds, aiming for a presentation that is systematic and emphasising how to enrich their structure "layer by layer".

\subsection{Topological manifolds}

The most basic level is the topological one. A manifold is a set of points $\mathcal{M}$ s.t. $\forall p \in \mathcal{M}, \exists$ an open set $U \in \mathcal{M}$ around $p$ and a map $\phi: U \rightarrow \mathbb{R}^{n}, p \mapsto \phi(p)=x^{\mu}$. Here $n$ is the dimension of $\mathcal{M}$, and $x^{\mu}$ is the coordinate of $p$. The pair $\{U, \phi\}$ is a (coordinate) chart.

A covering of $\mathcal{M}$ is a collection of open sets $\left\{U_{i}\right\}_{i \in I \subset \mathbb{N}}$ s.t. $\bigcup_{i \in I} U_{i}=\mathcal{M}$. The collection $\left\{U_{i}, \phi_{i}\right\}_{i \in I \subset \mathbb{N}}$ is an atlas of $\mathcal{M}$. On any non-empty intersections $U_{i} \cap U_{j} \neq \emptyset$, the transition functions are the maps $\phi_{j} \circ \phi_{i}^{-1}: \mathbb{R}^{n} \rightarrow \mathbb{R}^{n}$. A topological manifold $\mathcal{M}$ has continuous transition functions.

$\boldsymbol{R} \boldsymbol{m} \boldsymbol{k}$ : At this level, one defines the simplicial chain complex $\left(C_{k}, \partial_{k}\right)$, where $c_{k} \in C_{k}$ is a $k$-simplex - i.e. a $k$-dimensional polytope which is the convex envelope of its vertices - in $\mathcal{M}$, and $\partial$ is the boundary operator $-\partial_{k} c_{k}$ is the boundary of $c_{k}$. Since a boundary has no boundary, $\partial^{2} \equiv 0$. Cycles are $\partial$-closed simplices $(\in Z)$, boundaries are $\partial$-exact simplices $(\in B)$, and the associated simplicial homology $H_{\bullet}(\partial)=\bigoplus_{k=0}^{n} H_{k}(\partial)$ encodes informations about the topology of the manifold $\mathcal{M}$. 


\subsection{Differential manifolds}

A differentiable manifold have differentiable transition functions. If these are of class $C^{r}\left(\mathbb{R}^{n}\right)$, i.e. differentiable $r$ times, then we have a $C^{r}$-manifold. It the transition function are smooth, or of class $C^{\infty}\left(\mathbb{R}^{n}\right)$, the manifold is said smooth, or a $C^{\infty}$-manifold. There are several constructions on differentiable manifolds, stemming canonically from their differentiable structure.

The most elementary is its algebra of $\mathbb{R}$-valued functions, $f: \mathcal{M} \rightarrow \mathbb{R}$, equipped with the pointwise product $(f g)(p)=f(p) g(p)$. Denote $C^{r}(\mathcal{M})$ the algebra of functions $r$ times continuously differentiable, and $C^{\infty}(\mathcal{M})$ the algebra of smooth functions. In the following we will generically assume all maps/functions are smooth.

Directional differentiation of parametrised curves in $\mathcal{M}$ leads to the definition of vectors tangent to these curves, and more generally to the definition of the vector space $T_{x} \mathcal{M}$ of tangent vectors at a point $x \in \mathcal{M}$. The collection of all of them is the tangent bundle of $\mathcal{M}, T \mathcal{M}:=\bigcup_{x \in \mathcal{M}} T_{x} \mathcal{M}$. It is the most basic example of a vector bundle: the space attached to points of $\mathcal{M}$, called a fiber, is a vector space. The cotangent space $T_{x}^{*} \mathcal{M}$ at $x$ is the dual of $T_{x} \mathcal{M}$, and the cotangent bundle is $T^{*} \mathcal{M}:=\bigcup_{x \in \mathcal{M}} T_{x}^{*} \mathcal{M}$. Obviously, it is also a vector bundle.

Given a coordinate system $\left\{x^{\mu}\right\}$ on $U \subset \mathcal{M}$, at $x \in U$ partial derivatives $\left\{\partial_{\mu}\right\}$ constitute a basis of $T_{x} \mathcal{M}$, so that $X_{\mid x}=X^{\mu}(x) \partial_{\mu} \in T_{x} \mathcal{M}$. The dual basis of $T^{*} \mathcal{M}$ is $\left\{d x^{\mu}\right\}$, so that $\omega_{\mid x}=\omega_{\mu}(x) d x^{\mu} \in T_{x}^{*} \mathcal{M}$. By definition $d x^{\mu}\left(\partial_{\nu}\right)=\delta_{v}^{\mu}$, so $\omega_{\mid x}\left(X_{\mid x}\right)=\omega_{\mu}(x) X^{\mu}(x) \in \mathbb{R}$.

A vector field is a map $X: \mathcal{M} \rightarrow T \mathcal{M}, x \mapsto X_{\mid x}$. It is a section of $T \mathcal{M}$, and the set of such sections is noted $\Gamma(T \mathcal{M})$ - so that we write $X \in \Gamma(T \mathcal{M})$. Vector fields are derivations of $C^{\infty}(\mathcal{M})$. Under the bracket $[]:, \Gamma(T \mathcal{M}) \times \Gamma(T \mathcal{M}) \rightarrow \Gamma(T \mathcal{M}),(X, Y) \mapsto[X, Y], \Gamma(T \mathcal{M})$ is a Lie algebra.

In the same way, a covector field (a field of covectors) is a map $\omega: \mathcal{M} \rightarrow T^{*} \mathcal{M}, x \mapsto \omega_{\mid x}$, which is a section of $T^{*} \mathcal{M}: \omega \in \Gamma\left(T^{*} \mathcal{M}\right)$. It is also called a differential 1-form, i.e. it is a field of exterior 1 -forms of $T_{x} \mathcal{M}$. $\mathbb{R}$-valued differential 1-forms on $\mathcal{M}$ are noted $\Omega^{1}(\mathcal{M}, \mathbb{R})$. Thus $\Omega^{1}(\mathcal{M}, \mathbb{R})=\Gamma\left(T^{*} \mathcal{M}\right)$. A differential 1 -form evaluated on a vector field gives a $\mathbb{R}$-valued (smooth) function on $\mathcal{M}: \omega(X)=\omega_{\mu} X^{\mu} \in C^{\infty}(\mathcal{M})$. The evaluation operation is called the interior product, and is noted $\iota_{X} \omega=\omega(X)$.

By generalisation, a differential $k$-form is a map $\omega: \mathcal{M} \rightarrow \Lambda^{k}\left(T_{x} \mathcal{M}, \mathbb{R}\right), x \mapsto \omega_{\mid x}$, i.e. a field of exterior $k$-forms of $T_{x} \mathcal{M}$. One notes $\Omega^{k}(\mathcal{M}, \mathbb{R})$ the vector space of differential $k$-forms, and $\Omega^{\bullet}(\mathcal{M}, \mathbb{R})=\bigoplus_{k=0}^{n} \Omega^{k}(\mathcal{M}, \mathbb{R})$ the graded vector space of differential forms of $\mathcal{M}$. Notice that $\Omega^{0}(\mathcal{M}, \mathbb{R})=C^{\infty}(\mathcal{M})$. Equipped with an exterior product $\wedge: \Omega^{a}(\mathcal{M}, \mathbb{R}) \times \Omega^{b}(\mathcal{M}, \mathbb{R}) \rightarrow \Omega^{a+b}(\mathcal{M}, \mathbb{R})$ defined as in eq.(1), $\Omega^{\bullet}(\mathcal{M}, \mathbb{R})$ is a graded commutative algebra. The wedge product is also defined on algebra-valued differential forms, so $\Omega^{\bullet}(\mathcal{M}, A)$ is a graded algebra. There is no product on vector space-valued differential forms, so $\Omega^{\bullet}(\mathcal{M}, E)$ is simply a graded vector space.

Given $X \in \Gamma(T M)$ the interior product is $\iota_{X}: \Omega^{k}(\mathcal{M}, \mathbb{R}) \rightarrow \Omega^{k-1}(\mathcal{M}, \mathbb{R}), \omega \mapsto \iota_{X} \omega=$ $\omega(X, \cdots)$. Clearly $\iota_{X}{ }^{2} \equiv 0$ by the basic alternating property of forms. Furthermore, given the definition of the wedge product, it satisfies a graded Leibniz rule: $\iota_{X}(\alpha \wedge \beta)=\iota_{X} \alpha \wedge \beta+(-)^{a} \alpha \wedge \iota_{X} \beta$. The interior product is thus an anti-derivation of $\Omega^{\bullet}(\mathcal{M}, \mathbb{R})$. 
Another central anti-derivation of $\Omega^{\bullet}(\mathcal{M}, \mathbb{R})$ is the exterior - or de Rham - derivative, giving rise to the de Rham complex $\left(\Omega^{k}(\mathcal{M}, \mathbb{R}), d\right)$ :

$$
\cdots \longrightarrow \Omega^{k-1}(\mathcal{M}, \mathbb{R}) \stackrel{d}{\longrightarrow} \Omega^{k}(\mathcal{M}, \mathbb{R}) \stackrel{d}{\longrightarrow} \Omega^{k+1}(\mathcal{M}, \mathbb{R}) \longrightarrow \cdots
$$

with the associated de Rham chomology $H^{\bullet}(d)=\bigoplus_{k}^{n} H^{k}(d)$, where $H^{k}(d)=Z^{k}(d) / B^{k}(d)$ is the abelian group of $d$-closed but not $d$-exact differential $k$-forms on $\mathcal{M}$. The coordinate-free expression for the de Rham derivative is given by the Kozsul formula: for $\omega \in \Omega^{k}(\mathcal{M}, \mathbb{R})$,

$$
\begin{aligned}
d \omega\left(X_{1}, \cdots, X_{k+1}\right):=\sum_{i=1}^{k+1}(-)^{i+1} X_{i} \cdot \omega\left(X_{1}, \ldots, \hat{X}_{i}, \ldots, X_{k+1}\right) & \\
& +\sum_{i<j}(-)^{i+j+1} \omega\left(\left[X_{i}, X_{j}\right], \ldots, \hat{X}_{i}, \ldots, \hat{X}_{j}, \ldots X_{k+1}\right)
\end{aligned}
$$

The nilpotency property $d^{2} \equiv 0$ is ensured by the properties of the Lie bracket of vector fields (antisymmetry and Jacobi identity). For example:

- for $f \in \Omega^{0}(\mathcal{M}, \mathbb{R}), d f(X)=X(f)$,

- for $\omega \in \Omega^{1}(\mathcal{M}, \mathbb{R}), d \omega(X, Y)=X \cdot \omega(Y)-Y \cdot \omega(X)-\omega([X, Y])$.

In coordinate representation it is given by,

$$
d \omega=\frac{1}{k !} d \omega_{\mu_{1} \cdots \mu_{k}} d x^{\mu_{1}} \wedge \cdots \wedge d x^{\mu_{k}}=\frac{1}{(k+1) !} \partial_{[\mu} \omega_{\left.\mu_{1} \cdots \mu_{k}\right]} d x^{\mu} \wedge d x^{\mu_{1}} \wedge \cdots \wedge d x^{\mu_{k}} .
$$

The nilpotency property stems from the fact that $\partial_{\mu} \partial_{\nu}$ is symmetric while $d x^{\mu} \wedge d x^{\nu}$ is antisymmetric. For example:

- for $f \in \Omega^{0}(\mathcal{M}, \mathbb{R}), d f=\partial_{\mu} f d x^{\mu}$,

- for $\omega \in \Omega^{1}(\mathcal{M}, \mathbb{R}), d \omega=\frac{1}{2} \partial_{[\mu} \omega_{v]} d x^{\mu} \wedge d x^{\nu}$.

It is a useful exercise to check on a 0 -form $f$ and a 1 -form $\omega$ that eq.(11) and eq.(10) do coincide. The graded differential algebra $\left(\Omega^{\bullet}(\mathcal{M}, \mathbb{R}), d\right)$ is a canonical structure on a differential manifold $\mathcal{M}$.

Since $d, \iota_{X} \in \operatorname{Der}\left(\Omega^{\bullet}(\mathcal{M}, \mathbb{R})\right)$ are anti-derivations of degree 1 and -1 respectively, and since $\operatorname{Der}\left(\Omega^{\bullet}(\mathcal{M}, \mathbb{R})\right)$ is a (graded) Lie algebra, one defines the Lie derivative of a differential form along a vector field $X \in \Gamma(T \mathcal{M})$ as the degree 0 derivation $L_{X}:=\left[\iota_{x}, d\right]=\iota_{x} d+d \iota_{X} \in \operatorname{Der}\left(\Omega^{\bullet}(\mathcal{M}, \mathbb{R})\right)$ - a relation otherwise known as Cartan's formula. ${ }^{1}$ One shows that $\left[L_{X}, \iota_{Y}\right]=\iota_{[X, Y]}$, so that $\left[L_{X}, L_{Y}\right]=L_{[X, Y]}$ - i.e. the Lie derivative is a Lie algebra morphism.

The natural transformation group of a differentiable manifold is its group of diffeomorphisms, $\operatorname{Diff}(\mathcal{M})=\left\{\phi: \mathcal{M} \rightarrow \mathcal{M} \mid \phi \& \phi^{-1}\right.$ are bijective \& smooth (or $r$-differentiable) $\}$. It is a group under composition: for $\phi, \phi^{\prime} \in \operatorname{Diff}(\mathcal{M}), \phi \circ \phi^{\prime} \in \operatorname{Diff}(\mathcal{M})$. It acts on:

- $\Gamma(T M)$ by pushforward: $\phi_{*}: T_{x} \mathcal{M} \rightarrow T_{\phi(x)} \mathcal{M}, X_{\mid x} \mapsto\left(\phi_{*} X\right)_{\mid \phi(x)}$.

- $\Omega^{\bullet}(\mathcal{M}, \mathbb{R})$ by pullback: $\phi^{*}: \Lambda^{\bullet}\left(T_{\phi(x)} \mathcal{M}, \mathbb{R}\right) \rightarrow \Lambda^{\bullet}\left(T_{x} \mathcal{M}, \mathbb{R}\right), \omega_{\mid \phi(x)} \mapsto\left(\phi^{*} \omega\right)_{\mid x}$.

These are related operationally by: $\left(\phi^{*} \omega\right)_{\mid x}\left(X_{\mid x}\right)=\omega_{\mid \phi(x)}\left(\left(\phi_{*} X\right)_{\mid \phi(x)}\right)$. We have furthermore the naturality of the pullback: $\phi^{*} \circ d=d \circ \phi^{*}$.

\footnotetext{
${ }^{1}$ After the french mathematician Élie Cartan, the greatest differential geometer of the first half of the XXth century, whose name is also associated to e.g. Cartan's structure equation and Cartan connections - see section 4.2. His son Henri Cartan was also a famous and brilliant mathematician.
} 
The coordinate representation of the action of $\operatorname{Diff}(M)$ on a geometric object is formally indistinguishable from a mere change of coordinate representation of this geometric object. In the physics literature, the former is often called active diffeomorphisms, the latter passive diffeomorphisms. The interpretive difference between these is at the heart of the famous "hole argument", and reveals a deep physical teaching of General Relativity: points of $\mathcal{M}$ are unphysical [38].

One can enrich a differential manifold by equipping it with various non-canonical structures: - endow the algebra of functions of $\mathcal{M}$ with a Poisson bracket, and obtain a Poisson manifold, - endow $\mathcal{M}$ with a symplectic 2 -form (closed and non-degenerate), and obtain a symplectic manifold. A symplectic 2-form induces a Poisson bracket, so a symplectic manifold is a Poisson manifold. For the present concern, we will focus on the cases where one:

- endows $\mathcal{M}$ with a connection (1-form), so as to obtain a connection manifold, - endows $\mathcal{M}$ with a metric (tensor), so as to obtain a metric manifold.

If a manifold has both connection and metric, one can require compatibility of the two structures.

$\boldsymbol{R} \boldsymbol{m} \boldsymbol{k}$ : The topology of $\mathcal{M}$ can sometimes prevent the existence of "richer" structure on it. For example, not any compact differentiable manifold admit a Lorentzian metric (with signature +- -or -+++ ), only those whose Euler characteristic (which counts the number of "holes") vanishes. Another noticeable example: not all manifolds admit a spin structure, thus spinor fields, only those with vanishing second Stiefel-Whitney class (a topological invariant) do [39].

\subsection{Manifolds with connections}

Two vectors $X_{\mid x} \in T_{x} \mathcal{M}$ and $Y_{\mid x^{\prime}} \in T_{x^{\prime}} \mathcal{M}$ anchored at different points of $\mathcal{M}$ cannot be compared as they belong to different vector spaces that are not canonically related. To remedy the situation, one needs to add a new, non-canonical, structure on the differential manifold $\mathcal{M}$ that allows to "connect" distinct tangent spaces: a connection.

An infinitesimal connection is a map $\nabla: \Gamma(T \mathcal{M}) \times \Gamma(T \mathcal{M}) \rightarrow \Gamma(T \mathcal{M}),(X, Y) \mapsto \nabla_{X} Y$ which is: - $C^{\infty}(\mathcal{M})$-linear in its first argument, i.e. $\nabla_{f X} Y=f \nabla_{X} Y$ for $f \in C^{\infty}(\mathcal{M})$,

- additive and Leibniz-like in its second argument: $\nabla_{X}(f Y)=X(f) Y+f \nabla_{X} Y$.

In a coordinate system $\left\{x^{\mu}\right\}$ on $U \subset \mathcal{M}$, the symbol of the connection $\nabla$ is $\Gamma^{\alpha}{ }_{\mu \nu} \partial_{\alpha}:=\nabla_{\partial_{\mu}} \partial_{\nu}$. By the above two axioms, this implies $\nabla_{X} Y=X^{\mu}\left(\partial_{\mu} Y^{\alpha}+\Gamma_{\mu \nu}^{\alpha} Y^{\nu}\right) \partial_{\alpha}=: X^{\mu} \nabla_{\mu} Y^{\alpha} \partial_{\alpha}$, which gives the well-known coordinate representation of the "covariant derivative" of a vector field. ${ }^{2}$

The latter result can be recast in terms of differential forms: the components of the vector field $\nabla_{X} Y$ can be written as

$$
\left(\partial_{\mu} Y^{\alpha}+\Gamma_{\mu \nu}^{\alpha} Y^{\nu}\right) d x^{\mu}\left(X^{\lambda} \partial_{\lambda}\right)=\left(d Y^{\alpha}+\Gamma^{\alpha}{ }_{\nu} Y^{\nu}\right)(X)=:\left(\nabla Y^{\alpha}\right)(X)=\iota_{X} \nabla Y^{\alpha}
$$

The operator $\nabla:=d+\Gamma$ is the exterior covariant derivative acting on vectors, and $\Gamma$ is a matrixvalued 1-form, $\Gamma \in \Omega^{1}(\mathcal{M}, \mathbb{R}) \otimes \mathrm{M}(n)$, so $\Gamma=\Gamma^{\alpha}{ }_{\nu}=\Gamma^{\alpha}{ }_{\mu \nu} d x^{\mu}$, called the connection 1-form.

\footnotetext{
${ }^{2}$ The action of $\nabla_{X}$, for some $X \in \Gamma(T \mathcal{M})$, on 1-forms $\omega$ is obtained by requiring the Leibniz-like rule: $\nabla_{X}(\omega(Y))=$ $\left(\nabla_{X} \omega\right)(Y)+\omega\left(\nabla_{X} Y\right)$.
} 
Given a connection $\nabla$, the notion of (infinitesimal) transport of a vector field $X$ along a curve $c: \mathbb{R} \rightarrow \mathcal{M}, \tau \mapsto c(\tau)$, with tangent vector field $\dot{c}=\frac{d}{d \tau}=\frac{d c^{\mu}}{d \tau} \partial_{\mu}=\dot{c}^{\mu} \partial_{\mu}$, is defined as $\nabla_{\dot{c}} X$. The condition $\nabla_{\dot{c}} X=0$ defines the parallel transport of a vector field along $c$, i.e. vectors $X_{\mid c(\tau)}$ at different point $c(t)$ are declared parallel iff the condition holds. This gives a prescription to compare vectors $X_{\mid x} \in T_{x} \mathcal{M}$ and $Y_{\mid x^{\prime}} \in T_{x^{\prime}} \mathcal{M}$ : one needs only to provide a curve joining $x$ and $x^{\prime}$ along which to parallel transport $X_{\mid x}$ at $x^{\prime}$ where it is compared to $Y \mid x^{\prime}$.

This also allows to define geodesics $\gamma$ of $\mathcal{M}$ as auto-parallel curves, i.e. satisfying $\nabla_{\dot{\gamma}} \dot{\gamma}=0$. In coordinates, since $\dot{\gamma}=\dot{\gamma}^{\mu} \partial_{\mu}$, the above condition reads $\ddot{\gamma}^{\alpha}+\Gamma^{\alpha}{ }_{\mu \nu} \dot{\gamma}^{\mu} \dot{\gamma}^{\nu}=0$, which is the wellknown geodesic equation. The latter is both a generalisation of the principle of inertia (Newton's first law), and the coupling equation of point matter with gravity in GR [40].

The curvature of $\nabla$ is defined as the map $R: \Gamma(T \mathcal{M}) \times \Gamma(T \mathcal{M}) \times \Gamma(T \mathcal{M}) \rightarrow \Gamma(T \mathcal{M})$, $(X, Y, Z) \mapsto R(X, Y) Z:=\left(\left[\nabla_{X}, \nabla_{Y}\right]-\nabla_{[X, Y]}\right) Z$. It is clearly antisymmetric in its first two arguments. In a coordinate system $\left\{x^{\mu}\right\}$ on $U \subset \mathcal{M}$, its components are defined as

$$
R_{\beta, \mu \nu}^{\alpha} \partial_{\alpha}:=R\left(\partial_{\mu}, \partial_{\nu}\right) \partial_{\beta}=\left(\partial_{\mu} \Gamma_{\nu \beta}^{\alpha}-\partial_{\nu} \Gamma_{\mu \beta}^{\alpha}+\Gamma_{\mu \lambda}^{\alpha} \Gamma_{\nu \beta}^{\lambda}-\Gamma_{\nu \lambda}^{\alpha} \Gamma_{\mu \beta}^{\lambda}\right) \partial_{\alpha}
$$

and $R_{\beta, \mu v}^{\alpha}$ is the Riemann-Christoffel curvature tensor. The latter can be seen as the components of a matrix-valued differential 2-form $R \in \Omega^{2}(\mathcal{M}, \mathbb{R}) \otimes \mathrm{M}(n), R=R_{\beta}^{\alpha}=\frac{1}{2} R_{\beta, \mu \nu}^{\alpha} d x^{\mu} \wedge d x^{\nu}$. It is by the way easy to see that $R=d \Gamma+{ }^{1} / 2[\Gamma, \Gamma]$, which is an instance of Cartan's structure equation see section 4.3. So, $R$ is the curvature 2-form of the connection 1-form $\Gamma$.

The torsion of $\nabla$ is the antisymmetric map $T: \Gamma(T \mathcal{M}) \times \Gamma(T \mathcal{M}) \rightarrow \Gamma(T \mathcal{M})$, defined as $(X, Y) \mapsto T(X, Y):=\nabla_{X} Y-\nabla_{Y} X-[X, Y]$. Its coordinate components are given by

$$
T_{\mu \nu}^{\alpha} \partial_{\alpha}:=T\left(\partial_{\mu}, \partial_{\nu}\right)=\nabla_{\partial_{\mu}} \partial_{\nu}-\nabla_{\partial_{\mu}} \partial_{\mu}=\left(\Gamma_{\mu \nu}^{\alpha}-\Gamma_{\nu \mu}^{\alpha}\right) \partial_{\alpha}=\frac{1}{2} \Gamma_{[\mu \nu]}^{\alpha} \partial_{\alpha}
$$

which also define a vector-valued 2-form $T \in \Omega^{2}(\mathcal{M}, \mathbb{R}) \otimes \mathbb{R}^{n}, T=T^{\alpha}=\frac{1}{2} T^{\alpha}{ }_{\mu \nu} d x^{\mu} \wedge d x^{\nu}$. When $\nabla$ is torsion-free, $T \equiv 0$, its symbols - the components of $\Gamma$ - are symmetric $\Gamma^{\alpha}{ }_{\mu \nu}=\Gamma_{\nu \mu}^{\alpha}$. Torsion has been introduced into differential geometry by É. Cartan in the 1920's.

A manifold $\mathcal{M}$ can be Riemann-flat, i.e. $R \equiv 0$, yet still have non-vanishing torsion. This kind of spaces were studied by Einstein in the late 1920's when he worked on his attempts to unify gravity and electromagnetism (see the Einstein-Cartan correspondence [41]). If $\mathcal{M}$ is Cartan-flat, i.e. $R \equiv 0$ and $T \equiv 0$, then $\mathcal{M}=\mathbb{R}^{n}$ and the connection is trivial: all tangent spaces across the manifold are canonically isomorphic, ${ }^{3}$ the parallel transport between two points is independent of a choice of curve joining them.

\subsection{Metric manifolds}

A priori, on a differential manifold $\mathcal{M}$, endowed with a connection or not, there is no canonical means to define the modulus of a vector $X_{\mid x} \in T_{x} \mathcal{M}$, and by extension the length of a curve or the distance between two points. To do this, one needs a new, non-canonical, structure on $\mathcal{M}$ : a metric.

${ }^{3}$ And isomorphic to $\mathbb{R}^{n}=\mathcal{M}$, which means that one pays no price in pretending that vectors (fields) belong to $\mathcal{M}$ instead of $T \mathcal{M}$, as we are used to in elementary geometry. 
A (non-degenerate) metric field is a bilinear symmetric map $g: \Gamma(T \mathcal{M}) \times \Gamma(T \mathcal{M}) \rightarrow C^{\infty}(\mathcal{M})$, $(X, Y) \mapsto g(X, Y)$. That is, at any given $x \in \mathcal{M}, g_{\mid x}: T_{x} \mathcal{M} \times T_{x} \mathcal{M} \rightarrow \mathbb{R},\left(X_{\mid x}, Y_{\mid x}\right) \mapsto g_{\mid x}\left(X_{\mid x}, Y_{\mid x}\right)$, is a metric on the vector space $T_{x} \mathcal{M}$. Its coordinate components are defined as $g_{\mu \nu}:=g\left(\partial_{\mu}, \partial_{\nu}\right)$. So, by linearity $g(X, Y)=g_{\mu \nu} X^{\mu} Y^{\nu}$. In particular the norm of a vector field is $|X|^{2}:=g(X, X)=g_{\mu \nu} X^{\mu} X^{\nu}$. Clearly the metric can be written as $g=g_{\mu \nu} d x^{\mu} \otimes d x^{\nu}$.

If $g$ is positive definite, i.e. has signature $(r, s)=(n, 0)$, then $(\mathcal{M}, g)$ is a Riemannian manifold. In arbitrary signature $(r, s),(\mathcal{M}, g)$ is pseudo-Riemannian. An important subclass of the latter are Lorentzian manifolds, of signature $(r, s)=(n-1,1)$ : In GR, spacetime is modelled by (diffeomorphic classes of) such manifolds.

In the pseudo-Riemannian case, especially in the Lorentzian case, one says that a metric $g$ defines a causal structure on $\mathcal{M}$. Indeed, at every point $x \in \mathcal{M}$, it distinguishes space-like vectors s.t. $g_{\mid x}\left(X_{\mid x}, X_{\mid x}\right)>0$, time-like vectors s.t. $g_{\mid x}\left(X_{\mid x}, X_{\mid x}\right)<0$, and null - or light-like - vectors s.t. $g_{\mid x}\left(X_{\mid x}, X_{\mid x}\right)=0$ (the latter define the local light-cone at $x$ ). Accordingly, one defines space-like, time-like or null submanifolds (curves, surfaces...) whose tangent vectors are of the given type. This is the reason for the many distinctive features of Lorentzian geometry compared to Riemannian geometry.

Two further notable facts stems from the introduction of the metric $g$. First, it induces a Hodge duality on differential forms, $*_{g}: \Omega^{k}(\mathcal{M}, \mathbb{R}) \rightarrow \Omega^{n-k}(\mathcal{M}, \mathbb{R})$, defined as in eq.(3)-(4). Second, as $\mathcal{M}$ can be endowed with a connection $\nabla$, one can require that the choice of connection be "compatible" with the metric and satisfies $\nabla_{X} g(Y, Z)=g\left(\nabla_{X} Y, Z\right)+g\left(Y, \nabla_{X} Z\right)$. This condition ensures that if $X$ parallel transports $Y$ and $Z$, then their scalar product is covariantly constant along the flow of $X$. In particular, the norm of a parallelly transported vector is constant. This makes sense physically, as in GR the norm of the momentum vector of a point particle is its rest mass. There is a unique connection satisfying the further requirement $T=0$ : the Levi-Civita connection $\nabla=\nabla^{g}$. Taken together, the above two conditions imply that $\nabla^{g}$ is entirely determined by $g$ : its symbols are the well-known Christoffel symbols, $\Gamma^{\alpha}{ }_{\mu \nu}(g)$, expressed in terms of $g_{\mu \nu}$ and its first derivatives. The connection 1-form is then $\Gamma=\Gamma(g)$. As a result, the curvature 2-form $R$ also becomes a function of $g$ and its first and second derivatives.

The (pseudo-) length of a curve $c(\tau)$ with endpoints $x=c(0)$ and $x^{\prime}=c(1)$ is: $\ell(c):=$ $\int_{0}^{1} g(\dot{c}, \dot{c})^{1 / 2} d \tau$. Extremal curves $\gamma$ satisfy $\delta \ell(\gamma) \equiv 0,{ }^{4}$ which is in coordinates $\ddot{\gamma}^{\alpha}+\Gamma^{\alpha}{ }_{\mu \nu}(g) \dot{\gamma}^{\mu} \dot{\gamma}^{\nu}=0$. In other words, extremal curves are the geodesics of the Levi-Civita connection.

Conclusion: In this section, we have reviewed the hierarchy of four structure levels on a manifold, - the topological level: there is no differential calculus, but there is a simplicial complex $\left(C^{k}, \partial\right)$. - the differential level: $T \mathcal{M}$ is defined, so is the de Rham complex $\left(\Omega^{\bullet}(\mathcal{M}, \mathbb{R}), d\right)$, differential/variational calculus is possible.

- the connection level: it is possible to define parallel transport, geodesics, curvature, and torsion.

- the metric level: Hodge duality is defined on $\Omega^{\bullet}(\mathcal{M}, \mathbb{R})$, as well as the notion of (pseudo-) length and causal structure, and of LC connection, whose geodesics are extremal curves.

${ }^{4}$ They are of minimal length in the Riemanniann case, of maximal pseudo-length (proper time) in the Lorentzian case. This is why free-fall is the best course of action for someone who wants to maximise his lifespan after crossing the horizon of a black hole (don't fire your thrusters). 
Gravity happens on the last two levels, where are all the mathematical tools necessary to GR in its standard ("metric") formulation.

In the next section we review a far reaching way in which a manifold can be enriched. From this geometric perspective, spacetime is seen as a (remarkable) subspace of a larger space whose extra structures cast shadows that we perceive as gauge fields. This is the differential geometry of principal fiber bundles, which is the natural framework of classical gauge theories.

\section{Principal bundles and connections}

In sections 4.1 to 4.3 we review in a synthetic and self-contained manner many elementary definitions and facts about principal bundles, their associated bundles and the connections they can be endowed with. This gives us the kinematical and structural geometric data, so to speak, for gauge theories. In section 4.4 we sketch how gauge theories are constructed from these geometric data.

\subsection{Principal bundles and their smooth structure}

A principal bundle over a base $n$-dimensional manifold $\mathcal{M}$ is a smooth manifold $\mathcal{P}$ with a surjective projection $\pi: \mathcal{P} \rightarrow \mathcal{M}, p \mapsto \pi(p)=x$, supporting a smooth free right action by a Lie group $H, \mathcal{P} \times H \rightarrow \mathcal{P},(p, h) \mapsto R_{h} p:=p h$, and s.t. $\pi \circ R_{h}=\pi$. The Lie group $H$ is called the structure group of $\mathcal{P}$. The right action defines an equivalence relation on $\mathcal{P}$ : any $p, p^{\prime} \in \mathcal{P}$ such that $p^{\prime}=R_{h} p=p h$ belong to the same fiber $H_{x}$. Said otherwise, the fiber to which $p$ belongs is its $H$-orbit, so that $H_{x}$ and $H$ are homeomorphic as manifolds (hence the notation for the fiber), yet of course $H_{x}$ has no group structure.

A bundle is locally trivial: given $U \subset \mathcal{M}, \mathcal{P}_{\mid U}=U \times H$. A bundle (coordinate) chart is a smooth invertible map $\phi: \mathcal{P}_{\mid U} \rightarrow U \times H, p \mapsto(\pi(p), h)$, and a bundle atlas is $\left\{U_{i}, \phi_{i}\right\}_{i \in I \subset \mathbb{N}}$ where $\left\{U_{i}\right\}$ is a covering of $\mathcal{M}$. Locally it is always possible to find a section of $\pi$, called a trivialising or local - section $\sigma: U \rightarrow \mathcal{P}$, s.t. $\pi \circ \sigma=\mathrm{id}_{U}$. If there exists a global section $\sigma: \mathcal{M} \rightarrow \mathcal{P}$, then the bundle is trivial, $\mathcal{P}=\mathcal{M} \times H$. Given $\sigma_{i}$ and $\sigma_{j}$ sections over $U_{i}, U_{j} \subset \mathcal{M}$ s.t. $U_{i} \cap U_{j} \neq \emptyset$, on the overlap $\sigma_{j}=\sigma_{i} g_{i j}$ where $g_{i j}: U_{i} \cap U_{j} \rightarrow H$ is a transition function. The set $\left\{g_{i j}\right\}$ of transition functions associated to a covering $\left\{U_{i}\right\}_{i \in I \subset \mathbb{N}}$ of $\mathcal{M}$ are local data from which it is possible to reconstruct the bundle $\mathcal{P}$. Its possibly non-trivial topology is thus encoded into its transition functions.

The automorphism group of $\mathcal{P}$ is the subgroup of its diffeomorphisms that commute with the right $H$-action: $\operatorname{Aut}(\mathcal{P}):=\left\{\Psi \in \operatorname{Diff}(\mathcal{P}) \mid \Psi \circ R_{h}=R_{h} \circ \Psi\right\}$. It is the largest subgroup of $\operatorname{Diff}(\mathcal{P})$ preserving the fibration structure. Thus any $\Psi \in \operatorname{Aut}(\mathcal{P})$ projects to a well-defined $\psi=\pi \circ \Psi \in$ $\operatorname{Diff}(\mathcal{M})$. As the name suggest, $\operatorname{Aut}(\mathcal{P})$ is the natural transformation group of $\mathcal{P}$. The subgroup of vertical automorphisms further preserves the fibers, $\operatorname{Aut}_{v}(\mathcal{P}):=\{\Psi \in \operatorname{Aut}(\mathcal{P}) \mid \pi \circ \Psi=\pi\}$, its elements project to the identity transformations $\mathrm{id}_{x}$ on $\mathcal{M}$. It is easy to see that it is isomorphic to the gauge group $\mathcal{H}:=\left\{\gamma: \mathcal{P} \rightarrow H \mid \gamma(p h)=h^{-1} \gamma(p) h\right\}$, with the identification given by $\Psi(p)=p \gamma(p)$. We have the following SES of groups:

$$
0 \longrightarrow \operatorname{Aut}_{v}(\mathcal{P}) \simeq \mathcal{H} \stackrel{\iota}{\longrightarrow} \operatorname{Aut}(\mathcal{P}) \stackrel{\pi}{\longrightarrow} \operatorname{Diff}(\mathcal{M}) \simeq \operatorname{Aut}(\mathcal{P}) / \mathcal{H} \longrightarrow 0 .
$$

Without a splitting of this SES, one cannot lift a $\psi \in \operatorname{Diff}(\mathcal{M})$ into an element of $\operatorname{Aut}(\mathcal{P})$, or decompose uniquely a $\Psi \in \operatorname{Aut}(\mathcal{P})$ into a vertical automorphism and a diffeomorphism on $\mathcal{M}$. 
As a smooth manifold, $\mathcal{P}$ has a tangent bundle $T \mathcal{P}:=\bigcup_{p \in \mathcal{P}} T_{p} \mathcal{P}$ whose sections $\Gamma(T \mathcal{P})$ are vector field given in terms of their flow $\phi_{\tau}: \mathcal{P} \rightarrow \mathcal{P}$ by $X_{p}=\left.\frac{d}{d \tau} \phi_{\tau}(p)\right|_{\tau=0}$. A $H$-right invariant vector field $X \in \Gamma_{H}(T \mathcal{P})$ is s.t. $R_{h *} X_{p}=X_{p h}$, so that $\pi_{*} X_{p h}=\pi_{*} R_{h *} X_{p}=\pi_{*} X_{p}$ is a well defined vector fields $\widetilde{X} \in \Gamma(T \mathcal{M})$ on $\mathcal{M}$. Under the bracket [, ] of vector fields, $\Gamma_{H}(T \mathcal{P})$ is stable and therefore a Lie subalgebra of $\Gamma(T \mathcal{P})$. Then $\pi_{*}: \Gamma_{H}(T \mathcal{P}) \rightarrow \Gamma(T \mathcal{M})$ is a morphism of Lie algebras. Furthermore, it is easy to see that the flow $\phi_{\tau}$ of $X \in \Gamma_{H}(T \mathcal{P})$ is s.t. $\phi_{\tau} \circ R_{h}=R_{h} \circ \phi_{\tau}$, i.e. $\phi_{\tau} \in \operatorname{Aut}(\mathcal{P})$. In other words $\Gamma_{H}(T \mathcal{P})=\operatorname{LieAut}(\mathcal{P})$.

The right action of $H$ on $\mathcal{P}$ induces a canonical subbundle $V \mathcal{P} \subset T \mathcal{P}$. An element $X \in$ Lie $H$ gives a vertical vector $X_{p}^{v}:=\left.\frac{d}{d \tau} p e^{\tau X}\right|_{\tau=0}$ tangent to a curve $p e^{\tau X}$ contained in the fiber through $p$. The vector space of vertical vectors at $p$ is $V_{p} \mathcal{P} \subset T_{p} \mathcal{P}$, and the vertical subbundle is $V \mathcal{P}=\cup_{p \in \mathcal{P}} V_{p} \mathcal{P}$. Clearly $\pi_{*} X^{v}=0$, and one easily proves $R_{h *} X_{p}^{v}=\left(h^{-1} X h\right)_{p h}^{v}$. A vertical vector field is $X^{v} \in \Gamma(V \mathcal{P})$, and the map $\operatorname{Lie} H \rightarrow \Gamma(V \mathcal{P})$ is an injective morphism of Lie algebras. Any element of the Lie algebra of the gauge group Lie $\mathcal{H}:=\left\{\chi: \mathcal{P} \rightarrow \operatorname{Lie} H \mid \chi(p h)=h^{-1} \chi(p) h\right\}$ induces a $H$-right invariant vertical vector field via $\chi^{v}:=\left.\frac{d}{d \tau} p e^{\tau \chi}\right|_{\tau=0}$. The map Lie $\mathcal{H} \rightarrow \Gamma_{H}(V \mathcal{P})$ is a Lie algebra anti-isomorphism. ${ }^{5}$ Thus, corresponding to (15), we have the SES of Lie algebras

$$
0 \longrightarrow \Gamma_{H}(V \mathcal{P}) \simeq \operatorname{Lie} \mathcal{H} \stackrel{\iota}{\longrightarrow} \Gamma_{H}(T \mathcal{P}) \stackrel{\pi_{*}}{\longrightarrow} \Gamma(T \mathcal{M}) \simeq \Gamma_{H}(T \mathcal{P}) / \Gamma_{H}(V \mathcal{P}) \longrightarrow 0
$$

(also kown as the Atiyah Lie algebroid associated to $\mathcal{P}$ ). Here again, a splitting is needed to either lift a $X \in \Gamma(T \mathcal{M})$, or decompose uniquely $X \in \Gamma_{H}(T \mathcal{P})$ into a vertical vector field and a 'horizontal' part.

As a smooth manifold still, $\mathcal{P}$ has a de Rham complex $\left(\Omega^{\bullet}(\mathcal{P}), d\right)$, where $d$ is the de Rham, or exterior, derivative on $\mathcal{P}$. The interior product and Lie derivative are defined the usual way. An exterior product $\wedge$ is also defined the usual way on the space $\Omega^{\bullet}(\mathcal{P}, A)$ of differential forms with values in an algebra $(\mathrm{A}, \cdot)$, so that $\left(\Omega^{\bullet}(\mathcal{P}, \mathrm{A}), \wedge, \mathrm{d}\right)$ is a differential graded algebra. As we have already stressed, such a product is not defined on $\Omega^{\bullet}(\mathcal{P}, V)$ where $V$ is merely a vector space. But if $(V, \rho)$ is a representation for $H$, then one can define the vector space of equivariant forms on $\mathcal{P}$ as those for which the pullback by $R_{h}$ is well defined:

$$
\Omega_{\mathrm{eq}}^{\bullet}(\mathcal{P}, V)=\left\{\omega \in \Omega^{\bullet}(\mathcal{P}, V) \mid R_{h}^{*} \omega_{\mid p h}=\rho(h)^{-1} \omega_{\mid p},\right\} .
$$

Forms with trivial equivariance, $\Omega_{\text {inv }}^{\bullet}(\mathcal{P}, V)=\left\{\omega \in \Omega^{\bullet}(\mathcal{P}, V) \mid R_{h}^{*} \omega_{\mid p h}=\omega_{\mid p}\right.$, or $\left.L_{X^{v}} \omega=0\right\}$, constitute the subspace of invariant differential forms

The space of horizontal forms is $\Omega_{\text {hor }}^{\bullet}(\mathcal{P})=\left\{\omega \in \Omega^{\bullet}(\mathcal{P}) \mid \iota_{X^{v}} \omega=0\right\}$. A form which is both horizontal and equivariant is called tensorial:

$$
\Omega_{\text {tens }}^{\bullet}(\mathcal{P}, V)=\left\{\omega \in \Omega^{\bullet}(\mathcal{P}, V) \mid R_{h}^{*} \omega=\rho(h)^{-1} \omega \text {, and } \iota_{X^{v}} \omega=0, \forall X^{v} \in \Gamma(V \mathcal{P})\right\} .
$$

Notice that obviously $\Omega_{\text {tens }}^{0}(\mathcal{P}, V)=\Omega_{\text {eq }}^{0}(\mathcal{P}, V)$. Finally a form which is both horizontal and invariant is called basic:

$$
\Omega_{\text {basic }}^{\bullet}(\mathcal{P}, V)=\left\{\omega \in \Omega^{\bullet}(\mathcal{P}, V) \mid R_{h}^{*} \omega=\omega, \text { and } \iota_{X^{v}} \omega=0\right\} .
$$

${ }^{5}$ One shows that $\left[\chi^{v}, \eta^{v}\right]_{p}=(-[\chi, \eta])_{p}^{v}$. We have a Lie algebra isomorphism with the definition $\chi^{v}:=\left.\frac{d}{d \tau} p e^{-\tau \chi}\right|_{\tau=0}$. 
Basic forms are remarkable since, as their name suggests, they descend as well-defined forms on the base manifold $\mathcal{M}$. An equivalent definition of basic forms is that they belong to $\operatorname{Im}\left(\pi^{*}\right)$ :

$$
\Omega_{\text {basic }}^{\bullet}(\mathcal{P}, V)=\left\{\omega \in \Omega^{\bullet}(\mathcal{P}, V) \mid \exists \beta \in \Omega^{\bullet}(\mathcal{M}, V) \text { s.t. } \omega=\pi^{*} \beta\right\}
$$

The action of $\operatorname{Aut}_{v}(\mathcal{P}) \simeq \mathcal{H}$ on a differential form $\omega$ defines its active gauge transformation, $\omega^{\gamma}:=\Psi^{*} \omega$. Since we have on the one hand that $\Psi^{*} \omega_{\mid \Psi(p)}\left(X_{p}\right)=\omega_{\mid \Psi(p)}\left(\Psi_{*} X_{p}\right)$ for a generic $X \in \Gamma(T \mathcal{P})$, and on the other hand the well known result that for $\Psi \in \operatorname{Aut}_{v}(\mathcal{P})$,

$$
\Psi_{*} X_{p}=R_{\gamma(p) *} X_{p}+\left\{\gamma^{-1} d \gamma_{\mid p}\left(X_{p}\right)\right\}_{p \gamma(p)}^{v}=R_{\gamma(p) *}\left(X_{p}+\left\{d \gamma \gamma_{\mid p}^{-1}\left(X_{p}\right)\right\}_{p}^{v}\right)
$$

it is clear that the gauge transformation of a differential form is given by its equivariance and verticality properties. In particular, the gauge transformations of tensorial forms are immediate: $\omega \in \Omega_{\text {tens }}^{\bullet}(\mathcal{P}, V) \Rightarrow \omega^{\gamma}=\rho(\gamma)^{-1} \omega$. As a special case, basic forms are obviously gauge-invariant: $\omega \in \Omega_{\text {basic }}^{\bullet}(\mathcal{P}, V) \Rightarrow \omega^{\gamma}=\omega$. In more than one way, the latter are of paramount importance in gauge theories, as they encompass e.g. Lagrangians, action functionals, or candidate observables.

Given a trivialising section $\sigma$ over $U \subset \mathcal{M}$, a local representative of a form $\alpha$ on $\mathcal{P}$ is $a:=\sigma^{*} \alpha \in \Omega^{\bullet}(U)$. Local representatives $a^{\prime}=\sigma^{\prime *} \alpha$ and $a=\sigma^{*} \alpha$ on open sets $U^{\prime} \cap U \neq \emptyset$ must, on the overlap where $\sigma^{\prime}=\sigma g$, satisfy gluing relations often called passive gauge transformations. Since on the one hand $a_{\mid x}\left(X_{x}\right):=\sigma^{*} \alpha_{\mid \sigma(x)}\left(X_{x}\right)=\alpha_{\mid \sigma(x)}\left(\sigma_{*} X_{x}\right)$, for a generic $X \in \Gamma(T \mathcal{M})$, and on the other hand we have the well known result

$$
\sigma_{*}^{\prime} X_{x}=R_{g(x) *}\left(\sigma_{*} X_{x}\right)+\left\{g^{-1} d g_{\mid x}\left(X_{x}\right)\right\}_{\sigma^{\prime}(x)}^{v}=R_{g(x) *}\left(\sigma_{*} X_{x}+\left\{d g g_{\mid x}^{-1}\left(X_{x}\right)\right\}_{\sigma(x)}^{v}\right)
$$

it is again clear that the passive gauge transformation of the a local representative $a$ is determined by the equivariance and verticality properties of $\alpha$. It is in particular immediate that $\alpha \in \Omega_{\text {tens }}^{\bullet}(\mathcal{P}, V) \Rightarrow$ $a^{\prime}=\rho(g)^{-1} a$, and that $\alpha \in \Omega_{\text {basic }}^{\bullet}(\mathcal{P}, V) \Rightarrow a^{\prime}=a$. Again, for obvious reasons the latter are of special significance to gauge theories.

Passive gauge transformations are akin to coordinate changes on $\mathcal{M}$ (in GR), while active gauge transformations are akin to action by diffeomorphisms. Passive and active gauge transformations are formally alike, and locally indistinguishable. ${ }^{6}$

The notion of associated bundles is of special importance. Given a representation $(V, \rho)$ of $H$, define a right action of $H$ on $\mathcal{P} \times V$ by $((p, v), h) \mapsto\left(p h, \rho(h)^{-1} v\right)$, and consider the equivalence relation under this action $(p, v) \sim\left(p h, \rho(h)^{-1} v\right)$. One builds a (vector) bundle $E$ associated to $\mathcal{P}$ as the space of equivalence classes under this action, noted $E=\mathcal{P} \times{ }_{H} V:=\mathcal{P} \times V / \sim$, with projection $\pi_{E}(q)=\pi_{E}([p, v]):=\pi(p)=x \in \mathcal{M}$. The space $\Gamma(E)$ of sections of $E$ is isomorphic to the space $\Omega_{\mathrm{eq}}^{0}(\mathcal{P}, V)=\left\{\varphi: \mathcal{P} \rightarrow V \mid R_{h}^{*} \varphi=\rho(h)^{-1} \varphi\right\}$ of equivariant functions (0-forms) on $\mathcal{P}$. The correspondence is $\varphi \Rightarrow s(x):=[p, \varphi(p)] \sim[p h, \varphi(p h)]=\left[p h, \rho(h)^{-1} \varphi(p)\right]$. Sections of $E$, or equivariant functions (or rather their local representatives $\phi:=\sigma^{*} \varphi: U \rightarrow V$ ), represent various kinds of matter fields.

${ }^{6}$ Meaning that the pullback on $U$ by $\sigma$ of $\alpha^{\gamma}=\rho(\gamma)^{-1} \alpha$ cannot be told apart from $a^{\prime}=\rho(g)^{-1} a$. 


\subsection{Ehresmann connections}

All of the above come, freely, from the smooth structure of $\mathcal{P}$ (and the representations of its structure group). A keen observer may notice two "shortcomings" of this smooth structure.

First, one has no canonical way to compare a point $p \in H_{x}$ to a point $p^{\prime} \in H_{x^{\prime}}$. Or, said otherwise, there is no natural way to say to which point in $H_{x^{\prime}}$ does $p \in H_{x}$ "corresponds" to. This is an abstract version of the introductory problem of section $3.3,{ }^{7}$ a generalisation of the problem of parallel transport: One must give a (non-canonical) prescription on how to match points in adjacent fibers, i.e. given a curve $c(\tau) \in \mathcal{M}$ with $c(0)=x$ and $c(1)=x^{\prime}$, one must specify a correspondence - a curve in $\mathcal{P}$ - between $p \in H_{x}$ and its "parallel transport" $\widetilde{p} \in H_{x^{\prime}}$.

Secondly, the de Rham derivative does not preserve the space of tensorial forms, which have nice gauge transformations: $\omega \in \Omega_{\text {tens }}^{\bullet}(\mathcal{P}, V)$ but $d \omega \notin \Omega_{\text {tens }}^{\bullet}(\mathcal{P}, V)$ because $d \omega$ is not horizontal. This applies in particular, and is easily seen, for sections of associated bundles $\Gamma(E) \simeq \Omega_{\text {eq }}^{0}(\mathcal{P}, V)=$ $\Omega_{\text {tens }}^{0}(\mathcal{P}, V)$. This is a problems from a physical standpoint as this means we have no way to meaningfully differentiate matter fields, an observation that is the first act of the so-called gauge principle [42].

Both problems are solved by endowing $\mathcal{P}$ with non-canonical structure called a Ehresmann, or principal, connection 1-form. ${ }^{8}$ A connection 1-form is $A \in \Omega^{1}(\mathcal{M}, \mathrm{Lie} H)$ s.t.

$$
\begin{aligned}
& \text { i } R_{h}^{*} A_{\mid p h}=\operatorname{Ad}_{h^{-1}} A_{\mid p} \text {, i.e. } A \in \Omega_{\mathrm{eq}}^{1}(\mathcal{P}, \operatorname{Lie} H), \\
& \text { ii } A_{\mid p}\left(X_{p}^{v}\right)=X \in \operatorname{Lie} H \text {, where } X_{p}^{v} \in V_{p} \mathcal{P}
\end{aligned}
$$

The set $\mathcal{A}$ of Ehresmann connections on $\mathcal{P}$ is an affine space modelled on the vector space $\Omega_{\text {tens }}^{1}(\mathcal{P}$, Lie $H)$ : given $A^{\prime}, A \in \mathcal{A}$, it is easy to see that $A^{\prime}-A \in \Omega_{\text {tens }}^{1}(\mathcal{P}, \operatorname{Lie} H)$. Or, given $A \in \mathcal{A}$ and $\beta \in \Omega_{\text {tens }}^{1}(\mathcal{P}, \operatorname{Lie} H), A^{\prime}:=A+\beta \in \mathcal{A}$. One cannot add two connections.

Any connection $A \in \mathcal{A}$ splits the $\operatorname{SES}(16)$, because it is a retraction of the map $\iota: A \circ \iota=\operatorname{id}_{\text {Lie } \mathcal{H}}$. At any $p \in \mathcal{P}$, it allows to define a horizontal complement to $V_{p} \mathcal{P}$ in $T_{p} \mathcal{P}$ by $H_{p} \mathcal{P}:=\operatorname{ker} A_{\mid p}$, so that any $X_{p} \in T_{p} \mathcal{P}$ has horizontal component $X_{p}^{h}:=X_{p}-\left\{\omega_{\mid p}\left(X_{p}\right)\right\}_{p}^{v}$. A thus defines a non-canonical subbundle $H^{\mathcal{P}}=\cup_{p \in \mathcal{P}} H_{p} \mathcal{P} \subset T \mathcal{P}$. The horizontal lift of a curve $c(\tau)$ in $\mathcal{M}$ is a curve $c(\tau)^{h}$ in $\mathcal{P}$ whose tangent vector field is horizontal. This is the prescription we needed: $\widetilde{p}=c^{h}(1) \in H_{x^{\prime}}$ is the parallel transport of $p=c^{h}(0) \in H_{x}$. The horizontal lift of $\widetilde{X} \in \Gamma(\mathcal{M})$ is $\widetilde{X}^{h} \in \operatorname{ker} A$ s.t $\pi_{*} \widetilde{X}^{h}=\widetilde{X}$.

Furthermore, and most importantly for physics, a Ehresmann connection allows to define a covariant derivative $D^{A}: \Omega_{\mathrm{eq}}^{\bullet}(\mathcal{P}, V) \rightarrow \Omega_{\text {tens }}^{\bullet+1}(\mathcal{P}, V)$, which on $\Omega_{\text {tens }}^{\bullet}(\mathcal{P}, V)$ is given algebraically by $D^{A}=d+\rho_{*}(A)$. It is easy to show that $D^{A} \circ D^{A}=\rho_{*}(F)$, where $F$ is the curvature 2-form of $A$, given algebraically by Cartan's structure equation: $F=d A+1 / 2[A, A]$. By definition, $F \in \Omega_{\text {tens }}^{2}(\mathcal{P}, \operatorname{Lie} H)$, thus $D^{A}$ acts on it, trivially so, giving the Bianchi identity $D^{A} F=0$. From the above general discussion follows that its gauge transformation is $F^{\gamma}=\gamma^{-1} F \gamma$. Which can also be found via Cartan's structure equation and the fact that, due to the axioms i-ii and eq.(21), the gauge transformations of the connection is $A^{\gamma}=\gamma^{-1} A \gamma+\gamma^{-1} d \gamma$.

${ }^{7}$ Replace points $p, p^{\prime}$ in fibers $H_{x}$ and $H_{x^{\prime}}$ by vectors $X, Y$ in tangent spaces $T_{x} \mathcal{M}$ and $T_{x^{\prime}} \mathcal{M}$.

${ }^{8}$ Charles Ehresmann was a french mathematician, a towering figure of differential geometry, and a pupil of Élie Cartan. 
Locally, on $U \subset \mathcal{M}$, the local representative of $A$ via a section $\sigma$ is a Yang-Mills potential, while $\sigma^{*} F$ is the YM field strengh, and $\sigma^{*}\left(D^{A} \varphi\right)$ is the minimal coupling between the YM potential and a matter field $\phi$. Ehresmann connections are thus the geometric underpinning of (classical) Yang-Mills-Utiyama gauge theories.

\subsection{Cartan connections}

For gravitational gauge theories, another kind of connection is best suited: Cartan connections (see [33, 34]). Given $\operatorname{Lie} G \supset \operatorname{Lie} H$, a Cartan connection $\bar{A}$ on $\mathcal{P}$ is a $\operatorname{Lie} G$-valued 1-form s.t.

i $R_{h}^{*} \bar{A}_{\mid p h}=\operatorname{Ad}_{h^{-1}} \bar{A}_{\mid p}$, i.e. $\bar{A} \in \Omega_{\mathrm{eq}}^{1}(\mathcal{P}, \mathrm{Lie} G)$,

ii $\bar{A}_{\mid p}\left(X_{p}^{v}\right)=X \in \operatorname{Lie} H$, where $X_{p}^{v} \in V_{p} \mathcal{P}$,

iii $\forall p \in \mathcal{P}, \bar{A}_{\mid p}: T_{p} \mathcal{P} \rightarrow \operatorname{Lie} G$ is a linear isomorphism.

The set $\mathcal{B}$ of Cartan connections on $\mathcal{P}$ is an affine space modelled on the vector space $\Omega_{\text {tens }}^{1}(\mathcal{P}, \operatorname{Lie} G)$ : given $\bar{A}^{\prime}, \bar{A} \in \mathcal{B}$, it is easy to see that $\bar{A}^{\prime}-\bar{A} \in \Omega_{\text {tens }}^{1}(\mathcal{P}, \operatorname{Lie} G)$. Or, given $\bar{A} \in \mathcal{B}$ and $\beta \in$ $\Omega_{\text {tens }}^{1}(\mathcal{P}, \operatorname{Lie} G), \bar{A}^{\prime}:=\bar{A}+\beta \in \mathcal{B}$. Again, it makes no sense to add Cartan connections.

A pair $(\mathcal{P}, \bar{A})$ is a Cartan geometry. Contrary to an Ehresmann connection, a Cartan connection is not designed to split the sequence (16) and to define an horizontal subbundle $H \mathcal{P}$. Rather, the distinguishing axiom iii has several important consequences. First, obviously $\operatorname{dim} \mathcal{P}=\operatorname{dim} G$ and $\operatorname{dim} \mathcal{M}=\operatorname{dim} G / H$. Then, $\bar{A}$ induces a soldering on $\mathcal{M}$, i.e. due to $\operatorname{ker} \bar{A}=\emptyset$ one has the bundle isomorphism: $T \mathcal{M} \simeq \mathcal{P} \times_{H} \operatorname{Lie} G / \operatorname{Lie} H .{ }^{9}$

Relatedly, with $\tau: \operatorname{Lie} G \rightarrow \operatorname{Lie} G / \operatorname{Lie} H$ the projection, $e=e^{a}:=\tau(\bar{A}) \in \Omega_{\text {tens }}^{1}(\mathcal{P}, \operatorname{Lie} G / \operatorname{Lie} H)$ is a soldering form. Given a non-degenerate symmetric bilinear form $\eta=\eta_{a b}$ on $\operatorname{Lie} G / \operatorname{Lie} H$, a Cartan connection induces a metric on $U \subset \mathcal{M}$ via $g():,=\eta\left(\sigma^{*} e, \sigma^{*} e\right)$. In components, $\sigma^{*} e=$ $e^{a}{ }_{\mu} d x^{\mu} \in \Omega^{1}(U, \operatorname{Lie} G / \mathrm{Lie} H)$ and $g_{\mu \nu}=\eta_{a b} e^{a}{ }_{\mu} e^{b}{ }_{\nu}$. If $H$ preserves $\eta$, i.e. $\eta(h v, h w)=\eta(v, w)$, then $g$ is independent of $\sigma$ and $\mathcal{H}$-invariant, thus is well-defined across $\mathcal{M}$. Otherwise a gauge class $[g]$ may be induced (e.g. in conformal Cartan geometry $[g]$ is a conformal class) either passively by changing $\sigma$, or actively via the action of $\mathcal{H}$ on $\bar{A}$. On account of axioms i-ii and eq.(21), the latter is again given by $\bar{A}^{\gamma}=\gamma^{-1} \bar{A} \gamma+\gamma^{-1} d \gamma .{ }^{10}$ From these facts alone, one already appreciates how a Cartan geometry $(\mathcal{P}, \bar{A})$ reflects and encodes the geometry of $\mathcal{M}$, making it the right fit for (classical) gauge theories of gravity.

Consider a (left) (Lie $G, H)$-module $V$, i.e. a vector space $V$ supporting actions of $\operatorname{Lie} G$ via $\rho_{*}^{\prime}$ and of $H$ via $\rho$ which are s.t. $\rho_{* \mid \operatorname{Lie} H}^{\prime}=\rho_{*}$. Then, $\bar{A}$ defines a covariant derivative $D^{\bar{A}}:=d+\rho_{*}^{\prime}(\bar{A}): \Omega_{\text {eq }}^{\bullet}(\mathcal{P}, V) \rightarrow \Omega_{\text {tens }}^{\bullet+1}(\mathcal{P}, V)$ (acting in particular on $\Gamma(E)$ ). One finds again that $D^{\bar{A}} \circ D^{\bar{A}}=\rho_{*}^{\prime}(\bar{F})$, where $\bar{F}$ is the curvature of $\bar{A}$ defined via Cartan's structure equation, $\bar{F}:=d \bar{A}+1 / 2[\bar{A}, \bar{A}] \in \Omega_{\text {tens }}^{2}(\mathcal{P}, \operatorname{Lie} G)$, and satisfy a Bianchi identity $D^{\bar{A}} \bar{F}=0$. The torsion of the Cartan connection is $T:=\tau(\bar{F}) \in \Omega_{\text {tens }}^{2}(\mathcal{P}, \operatorname{Lie} G / \operatorname{Lie} H)$, a notion obviously non-existent for Ehresmann connections.

${ }^{9}$ If $\mathrm{Lie} H$ is a subalgebra of $\mathrm{Lie} G$ but not an ideal, $\mathrm{LieG} / \mathrm{LieH}$ is merely a vector space - not a subalgebra - and $H$ acts on it via the Ad representation, or simply by left multiplication, according to the situation. As for Lie $G$, it acts via the ad representation.

${ }^{10}$ Notice that there are no gauge transformations corresponding to the group $G$, or the Lie algebra Lie $G$ ! 
Constraints on $\bar{F}$ can be imposed so that $\bar{A}$ 's only degrees of freedom (d.o.f.) are those of its soldering form $e$. These normality conditions, which sets some components of $\bar{F}$ to 0 and most often comprise at least $T \equiv 0$, single out a unique normal Cartan connection. Up to gauge transformations that is: since $\bar{F} \in \Omega_{\text {tens }}^{2}(\mathcal{P}, \operatorname{Lie} G)$ one has still $\bar{F}^{\gamma}=\gamma^{-1} \bar{F} \gamma$, so that normality conditions (torsionlessness in particular) are preserved by the action of $\mathcal{H}$.

It may be noticed that $\bar{F}=0$ implies that the base manifold is a homogeneous space $\mathcal{M} \simeq G / H$. Indeed, the Lie group $G$ is a $H$-bundle over the homogeneous space $G / H$, and the Maurer-Cartan form $\theta \in \Omega^{1}(G, \operatorname{Lie} G)$, satisfying $d \theta+1 / 2[\theta, \theta]=0$, is an instance of flat Cartan connection. The pair $(G, \theta)$ is called a Klein geometry based on the Klein pair $(G, H) .{ }^{11}$ Standard Euclidean and Minkowskian geometries, are special cases of Klein geometries based on the pair $\left(S O(n) \ltimes \mathbb{R}^{n}, S O(n)\right)$ and $\left(S O(1, n-1) \ltimes \mathbb{R}^{n}, S O(1, n-1)\right)$ respectively. A Klein geometry $(G, \theta)$ is the "homogeneous model" generalised by a Cartan geometry $(\mathcal{P}, \bar{A})$. Flatness in the sense of Cartan therefore generalises flatness in the sense of (pseudo-) Riemannian geometry. We reproduce here the nice diagram of Sharpe [33] illustrating how Cartan geometry encompasses both Klein and Riemann geometries.

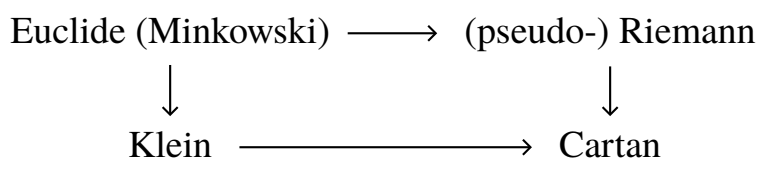

The subclass of reductive Cartan geometries is especially noteworthy: they are those for which there is a $\operatorname{Ad}(H)$-invariant decomposition $\operatorname{Lie} G=\operatorname{Lie} H+V^{n}$. This means we have a clean split of the Cartan connection as $\bar{A}=A+e$, where $A$ is a Ehresmann connection on $\mathcal{P}$. The curvature splits accordingly $\bar{F}=F+T$. Pseudo-Riemannian geometry, ${ }^{12}$ which is based on $\mathfrak{i s o}(r, s)=\mathfrak{s o}(r, s)+\mathbb{R}^{n}$, clearly belongs to this subclass.

Parabolic Cartan geometries are another remarkable subclass where one has a $|k|$-grading of $\operatorname{Lie} G$, i.e. $\operatorname{Lie} G=\bigoplus_{-k \leq i \leq k} \operatorname{Lie} G_{i}$ s.t. $\left[\operatorname{Lie} G_{i}, \operatorname{Lie} G_{i}\right] \subset \operatorname{Lie} G_{i+j}$, and $H$ is s.t. $\operatorname{Lie} H=$ $\bigoplus_{0 \leq i \leq k} \operatorname{Lie} G_{i}$. Both $\bar{A}$ and $\bar{F}$ split along the $|k|$-grading, and here also the Lie $H$-part of $\bar{A}$ is a Ehresmann connection. An important example is conformal Cartan geometry, which is based on the $|1|$-graded algebra $\mathfrak{s o}(r+1, s+1)=\mathbb{R}^{n} \oplus \mathfrak{c o}(r, s) \oplus \mathbb{R}^{n *}$, with $\mathfrak{c o}(r, s)=\mathfrak{s o}(r, s) \oplus \mathbb{R}$, and where $H$ is s.t. $\operatorname{Lie} H=\mathfrak{c o}(r, s) \oplus \mathbb{R}^{n *}$. The spin version in case $n=4$, based on the $|1|$-grading of $\mathfrak{s u}(2,2)$, is very closely related to twistor geometry $[43,44]$.

The local representatives on $\mathcal{M}$ of the Cartan connection $\bar{A}$ and its curvature $\bar{F}$ represent respectively the gravitational gauge potential and its curvature/field strength. Sections $\Gamma(\mathrm{S})=$ $\Omega^{0}(\mathcal{P}, S)$ of associated spinor bundles built via spin representations $S$ of $H$ describe spinorial matter fields. In both the above subclasses, the local representative of the covariant derivative induced by the Lie $H$-part of $\bar{A}$ acting on $\Gamma(S)$ represents the minimal coupling of matter fields to gravity.

\footnotetext{
${ }^{11}$ After the mathematician Felix Klein, who suggested in his 1872 Erlangen program to classify and study non-Euclidean homogeneous spaces via their transformation groups.

12 As reformulated by Cartan via his "moving frame", and independently by Einstein via his "vierbein/vielbein" or tetrad field, i.e. the soldering $e$.
} 


\subsection{Gauge theories: a recipe}

We are now ready to appreciate how, in the same way that gravity is the dynamics of the geometry of a metric and connection manifold $(\mathcal{M}, \nabla, g)$, a gauge theory is the dynamics of the geometry of a bundle $\mathcal{P}$ endowed with a connection.

\subsubsection{Yang-Mills-Utiyama gauge theories}

The recipe to cook a gauge theory is quite simple, it requires only two things. First, the geometric elements detailed in sections 4.1-4.3 provide the "kinematics", so to speak. The dynamics is given by a choice of Lagrangian (of action) functional, i.e. a smooth map $L: \mathcal{A} \times \Gamma(E) \rightarrow \Omega^{n}(\mathcal{P}, \mathbb{R})$, $(A, \varphi) \mapsto L(A, \varphi)$, where $n=\operatorname{dim} \mathcal{M}$.

To build such a map the needed toolkit is quite obvious. Since we want to build a $n$-form from forms of degree zero $(\varphi)$, one $(A)$ and two $(F)$, the wedge product and the Hodge dual operator may come handy. Since we want to build a $\mathbb{R}$-valued form out of Lie $H$-valued $(A, F)$ and $V$-valued $(\varphi)$ forms, we need non-degenerate bilinear - or multilinear - forms on Lie $H$ and $V$. The Killing form ${ }^{13} B: \operatorname{Lie} H \times \operatorname{Lie} H \rightarrow \mathbb{R}$ of a Lie algebra will do, which for classical Lie algebras with fundamental matrix representation is essentially the trace operator $B \propto \operatorname{Tr}$. On the vector space $V$, a scalar product $\langle\rangle:, V \times V \rightarrow \mathbb{R},(v, w) \mapsto\langle v, w\rangle$, is natural. The non-degeneracy requirement is not superfluous. Indeed, field equations are obtained via a variational principle, requiring $\delta L=E(\delta A, \delta \varphi ; A, \varphi)+d \theta=0$ with $E$ linear in $\delta A$ and $\delta \varphi$. The boundary term being often neglected, ${ }^{14} E(\delta A, \delta \varphi ; A, \varphi)=0 \forall \delta A, \delta \varphi$ gives the field equations for $A$ and $\varphi$ iff the nondegeneracy requirement holds. ${ }^{15}$

Even under such constraints, there is still important freedom in the choice of $L$. But for $L(A, \varphi)$ to descend as a well-defined top-form on $\mathcal{M}$, it must be basic, which in turn implies it is $\mathcal{H}$-invariant: $L\left(A^{\gamma}, \varphi^{\gamma}\right)=L(A, \varphi)$. The requirement that the Lagrangian be invariant under gauge transformations is known in physics as the gauge principle. ${ }^{16}$ For this, it is useful that the Killing form is $\operatorname{Ad}_{H}$-invariant, and that $H$ is unitary for the scalar product, i.e. $\langle h v, h w\rangle=\langle v, w\rangle \forall h \in H$. It is striking that almost all interesting or fundamental gauge theories are the simplest examples compatible with such elementary desiterata.

- Yang-Mills theory on a $n$-dimensional manifold is based on a bundle $\mathcal{P}(\mathcal{M}, H)$ with $H=S U(N)$ whose gauge group is $\mathcal{S U}(N)$, and is given by the Lagrangian $L_{\mathrm{YM}}(A)=\frac{1}{2} \operatorname{Tr}(F \wedge * F)$. Since $\delta F=D^{A}(\delta A)$, the field equations are easily found to be $D^{A} * F=0$, which are the famous Yang-Mills equations. Notice that gauge-invariance forbids a mass term $m^{2} \operatorname{Tr}(A \wedge * A)$ in the Lagrangian: the gauge symmetry implies that gauge potentials are massless.

\footnotetext{
${ }^{13}$ Introduced by Élie Cartan in his $1894 \mathrm{PhD}$ dissertation. While the Cartan matrix of a Lie algebra was introduced by Wilhelm Killing.

${ }^{14}$ It is yet a crucial object for the covariant Hamiltonian formalism (or covariant phase space methods) which aims at studying the symplectic structure of gauge field theory, and where $\theta$ is know as the presymplectic potential [45-48]. It is often used in classical and quantum gravity [49-51], and in the study of gauge field theory over bounded regions, relating e.g. to the notion of entanglement entropy, see e.g. [52, 53] or [54].

${ }^{15}$ Remark that as $\mathcal{A}$ is an affine space, $\delta A \in \Omega_{\text {tens }}^{1}(\mathcal{P}, \operatorname{Lie} H)$, so that $A^{\prime}=A+\delta A \in \mathcal{A}$.

${ }^{16}$ It is the standard heuristic behind the construction of gauge theories, and is understood as imposing a meaningful physical constraint on the choice of Lagrangian, quite in the same way that the general relativity/covariance principle is the heuristics leading to GR. To appreciate the historical advent of the gauge principle we recommend the book by O'Raifeartaigh [24].
} 
In the abelian case, $H=U(1)$ and $\mathcal{H}=\mathcal{U}(1)$, we get Maxwell's theory $L_{\mathrm{EM}}(A)=\frac{1}{2} F \wedge * F$, and the field equations $d * F=0$ are half of Maxwell's equations: the other - source-free - half is provided by the Bianchi identity $d F=0$. These come from the underlying geometry, not from the choice of Lagrangian, and are thus "kinematical"/"non-dynamical". The same can be said of the Bianchi identity $D F=0$ in the non-abelian case. Echoing the previous remark, the $\mathcal{U}(1)$ gauge symmetry "predicts" the photon $A$ to be massless.

In $n=4$ dimension, a self dual YM field is s.t. $* F= \pm F$, in which case the YM Lagrangian becomes a topological invariant (as is manifest by the disappearance of the Hodge star, i.e. of the metric, from the Lagrangian) known as the Chern class of the bundle $\mathcal{P}: L_{\mathrm{YM}}(A) \propto \operatorname{Tr}(F \wedge F)$. Clearly then, such a self dual YM field automatically satisfies the field equations - as these reduce to the Bianchi identity $D * F= \pm D F \equiv 0$ - and is known in physics as an instanton.

When a topological term is added to the Lagrangian of a theory, the classical field equations are not modified, but there can be other effects. ${ }^{17}$ Such a term e.g. contributes to the vacuum structure of QCD whose Lagrangian (omitting the matter sector) is $L_{\mathrm{QCD}}(A)=L_{\mathrm{YM}}(A)+\theta \operatorname{Tr}(F \wedge F)$ and is $\mathcal{S U}(3)$-invariant. The topological term, called the "theta term" of $\mathrm{QCD}$, is responsible for possible yet unobserved CP-breaking in strong interactions - an issue known as the "strong CP problem".

- Still considering a $S U(N)$-bundle $\mathcal{P}$ with connection $A$, given $\varphi \in \Omega_{\text {tens }}^{0}(\mathcal{P}, V)$ with covariant derivative $D^{A} \varphi \in \Omega_{\text {tens }}^{1}(\mathcal{P}, V)$, the Klein-Gordon Lagrangian $L_{\mathrm{KG}}(A, \varphi)=\left\langle D^{A} \varphi, * D^{A} \varphi\right\rangle-m^{2}\langle\varphi, * \varphi\rangle$ is clearly invariant under $\mathcal{S U}(N)$. The field equation is the $\mathrm{KG}$ equation $D^{A} * D^{A} \varphi+m^{2} * \varphi=0$, which describes the propagation of a massive scalar field of mass $m$. The massless case $m=0$ is a valid limit. Notice that it is geometrically impossible to built a Lagrangian of order less that 2 in the derivative of the field, as no pairing of (the Hodge duals of) a 0-form $(\varphi)$ and a 1-form $\left(D^{A} \varphi\right)$ can give a $n$-form.

If we have instead $V$-valued spinor fields $\psi \in \Omega_{\mathrm{eq}}^{0}(\mathcal{P}, S \otimes V)$, one defines the Clifford algebravalued 1-form $\gamma=\gamma_{\mu} d x^{\mu}=\gamma_{a} e^{a}{ }_{\mu} d x^{\mu}=\gamma_{a} e^{a}$, where $e^{a}$ is the soldering form and $\left\{\gamma_{a}\right\}$ are the Dirac gamma matrices satisfying $\gamma_{a} \gamma_{b}+\gamma_{b} \gamma_{a}=2 \eta_{a b}$, so that $\gamma_{\mu} \gamma_{\nu}+\gamma_{\nu} \gamma_{\mu}=2 g_{\mu \nu}$. The Dirac operator is then $\not^{A}:=\gamma \wedge * D^{A}: \Omega_{\mathrm{eq}}^{0}(\mathcal{P}, S \otimes V) \rightarrow \Omega_{\text {tens }}^{1}(\mathcal{P}, S \otimes V)$. It allows to built the 1 st-order Dirac Lagrangian $L_{\text {Dirac }}(A, \psi)=\left\langle\psi, \not D^{A} \psi\right\rangle-m\langle\psi, * \psi\rangle$, whose associated Dirac equation $\not D^{A} \psi-m * \psi=0$ describes the propagation of a massive spin $1 / 2$ fermion field.

- All of the above feature in the Lagrangian of the Standard Model of particle physics. For example, the prototypical form of the electroweak sector of the SM (omitting fermions) is

$$
L(A, \psi, \varphi)=\frac{1}{2} \operatorname{Tr}(F \wedge * F)+\left\langle D^{A} \varphi, * D^{A} \varphi\right\rangle-V(\varphi)
$$

The Higgs field $\varphi$ interacts with $A$ via minimal coupling $D^{A} \varphi$. This interaction generates a mass term for $A$ when the potential $V(\varphi)=\mu^{2}\langle\varphi, * \varphi\rangle+\lambda\langle\varphi, * \varphi\rangle^{2}(\lambda>0)$ is minimised by non-vanishing configurations $\left\{\varphi_{0}\right\}$ of $\varphi$ (whose existence depends on the sign of the parameter $\mu^{2}$ ).

\footnotetext{
${ }^{17}$ Notably it modifies the presymplectic potential, thus the symplectic structure, of the theory.
} 
Of course there are Lagrangians $L(A, \varphi)$ that are not basic, and thus not gauge-invariant, yet still of great interest in theoretical physics. A very notable example is Chern-Simons theory, which is a topological theory based on a $S U(N)$-bundle $\mathcal{P}$ over a $n=3$-dimensional $\mathcal{M}$, whose Lagrangian is $L_{\mathrm{CS}}(A)=\operatorname{Tr}\left(A d A+{ }^{2} / 3 A^{3}\right)$ with associated field equation $F=0$. The abelian CS theory is simply $L_{\mathrm{AbCS}}(A)=\operatorname{Tr}(A d A)$ with field equation $F=d A=0$. It is related to the Chern class by $\operatorname{Tr}(F \wedge F)=d L_{\mathrm{CS}}(A)$, which explains why the latter only affects the presymplectic potential of a Lagrangian and not its field equations.

\subsubsection{Gravitational gauge theories}

In section 3, we have commented that gravitational theories (starting with GR) "live" at the connection and metric level of a manifold. That is, gravitation $i s$ the geometry of spacetime, represented by (a $\operatorname{Diff}(\mathcal{M})$-class of $)(\mathcal{M}, \nabla, g)$. As we have seen in section 4.3, with Cartan the geometry of the gauge structure $(\mathcal{P}, \bar{A})$ is intimately related to that of $\mathcal{M}$. So Cartan geometry is the fitting framework for gauge formulations of gravity.

That is, a Cartan geometry provide the kinematics of a gauge gravitational theory, and its dynamics is given by a choice of Lagrangian $L: \mathcal{B} \times \Gamma(\mathrm{S}) \rightarrow \Omega^{n}(\mathcal{P}, \mathbb{R}),(\bar{A}, \psi) \mapsto L(\bar{A}, \psi)$ - if we consider gravity coupled to spinor fields. The construction of such a Lagrangian form is subjected to the same procedure and constraints previously sketched. In particular, one may require $L(\bar{A})$ to be basic, and thus $\mathcal{H}$-invariant: $L\left(\bar{A}^{\gamma}\right)=L(\bar{A})$. This is a gauge principle applied to gravitation. ${ }^{18}$ Let us consider in turn three examples.

- Consider the (reductive) Cartan geometry $(\mathcal{P}, \bar{A})$ based on $(G, H)=\left(S O(1,3) \ltimes \mathbb{R}^{4}, S O(1,3)\right)$, with gauge group $\mathcal{S} O(1,3)$. The Cartan connection splits as $\bar{A}=A+e$, where $A=A^{a}{ }_{b}$ is a Ehresmann connection (the spin connection) and the $\mathbb{R}^{4}$-valued $e=e^{a}$ is the soldering form (or cotetrad field). The curvature is $\bar{F}=R+T$, where $R=d A+{ }_{1}^{1 / 2}[A, A] \in \Omega_{\text {tens }}^{2}(\mathcal{P}$, LieSO $)$ is the Riemann curvature 2-form of $A$, and $T=D^{A} e=d e+A \wedge e$. If $\bar{F}=0$, the base manifold is the homogeneous model space: $\mathcal{M} \simeq G / H \simeq \mathbb{R}^{n}$. The normal Cartan connection is s.t. $\bar{F}=R$, which makes $A=A(e)$ the Levi-Civita connection.

We can write the Lagrangian of Einstein-Cartan gravity (or Kibble-Sciama gravity) as

$$
L_{\mathrm{EC}}(\bar{A})=L_{\mathrm{EC}}(A, e)=R \eta^{-1} \bullet e \wedge e^{T}:=R^{a b} e^{c} e^{d} \varepsilon_{a b c d}
$$

The field equations are $E(\delta \bar{A}, \bar{A})=\delta A \bullet D^{A} e \wedge e^{T}+\delta e \wedge e^{T} \bullet R \eta^{-1}=0$. The sectors of the Lie algebra are independent so we have $T=0$ enforcing the normality of the Cartan connection, and $R^{a b} e^{c} \varepsilon_{a b c d}=0$ are the vacuum Einstein field equations for the tetrad field ( $\sim$ the metric). Clearly the ground state of the theory is $R=0$, i.e. $\bar{F}=0$, that is precisely the homogeneous model of the Cartan geometry. We then recover GR in the tetrad formulation.

${ }^{18}$ The idea that a gauge principle allows to recover GR (in the Cartan-Einstein tetrad formulation) was first proposed by Ryoyu Utiyama, a japanese physicist, in the same 1956 paper in which he fully articulates the modern understanding of the gauge argument, and how to "gauge" a global symmetry (Lie) group to obtain a gauge theory. His work, completed in mid-1954, is independent of the $\mathcal{S U}(2)$ Yang-Mills paper issued the same year, and is much more general (the $\mathcal{S U}(2)$ case being yet again independently discovered by Ronald Shaw - a student of A. Salam - in 1954 and published in his $\mathrm{PhD}$ thesis). It is a great historical injustice that gauge theories are associated almost entirely to Yang and Mills, while Utiyama's far reaching contribution is seldom remembered. See again [24] for an accurate account of the events. 
If one adds the cosmological constant term $-\frac{\Lambda}{6} e \wedge e^{T} \bullet e \wedge e^{T}=-\frac{\Lambda}{6} e^{a} e^{b} e^{c} e^{d} \varepsilon_{a b c d}$ to $L_{\mathrm{EC}}(\bar{A})$ the $\delta e$ part of the field equations becomes $\left(R^{a b}-\frac{\Lambda}{3} e^{a} \wedge e^{b}\right) e^{c} \varepsilon_{a b c d}=0$. This time the ground state is $R=\frac{\Lambda}{3} e \wedge e^{T} \eta$, i.e. de Sitter (or anti-de Sitter) space, which is not the homogeneous model of the Cartan geometry. But there is an alternative framing of this theory.

- Consider the Cartan geometry $(\mathcal{P}, \bar{A})$ based on $(G, H)$ with $H=S O(1,3)$ and gauge group $\mathcal{S O}(1,3)$ still, but $G$ is either $\operatorname{SO}(1,4)(\Lambda>0)$ or $S O(2,3)(\Lambda<0)$, so that the model homogeneous space is the de Sitter or anti-de Sitter space $\simeq G / H \simeq(A) d S^{n}$. The Cartan connection splits as $\bar{A}=A+\frac{1}{\ell} e$, with $\frac{1}{\ell^{2}}=\frac{\Lambda}{3}$, and its curvature is $\bar{F}=F+\frac{1}{\ell} T$ with $F=R-\frac{1}{\ell^{2}} e \wedge e^{T} \eta \in \Omega_{\text {tens }}^{2}(\mathcal{P}$, Lie $H)$ and $T=D^{A} e$. Again, the normal Cartan connection is s.t. $\bar{F}=F$, so $A=A(e)$. If $\bar{A}$ is Cartan flat, $\bar{F}=0$, then the base spacetime manifold is the homogeneous space $\mathcal{M} \simeq(A) d S^{n}$.

The Lagrangian of McDowell-Mansouri gravity is

$$
\begin{aligned}
L_{\mathrm{MM}}(\bar{A})=L_{\mathrm{MM}}(A, e)=\frac{1}{2} F \bullet F & =\frac{1}{2} R \bullet R-\frac{1}{\ell^{2}}\left(R \eta^{-1} \bullet e \wedge e^{T}-\frac{1}{2 \ell^{2}} e \wedge e^{T} \bullet e \wedge e^{T}\right), \\
& =\frac{1}{2} R \bullet R-\frac{1}{\ell^{2}} L_{\mathrm{EC}}(\bar{A}) .
\end{aligned}
$$

The term $\frac{1}{2} R \bullet R$ is a topological invariant called the Euler density. Thus, the field equations are the same as those of $L_{\mathrm{EC}}(\bar{A})$, and their ground state now coincide with the homogeneous space of the Cartan geometry. The Euler density modifies the presymplectic potential of the theory, which improves the symplectic structure of $L_{\mathrm{MM}}$ compared to $L_{\mathrm{EC}}$.

In both the above examples, the spinors naturally associated to the Cartan geometry are $S L(2, \mathbb{C})$ Weyl or Dirac spinors describing fermionic fields that couple to gravity via the Dirac operator $\not D^{A} \psi$, and can be incorporated into the Lagrangians $L_{\mathrm{MM}}$ and $L_{\mathrm{EC}}$ by adding the Dirac term $L_{\text {Dirac }}$. In which case the field equations change: the spinor field $\psi$ act as a source for both the torsion (which does not propagate outside matter) and the curvature tensor $R$ via the Einstein field equations.

- As our last example, consider the conformal Cartan geometry $(\mathcal{P}, \bar{A})$, where $G=S O(2,4)$ is the conformal group of the compactified Minkowski space $\overline{\mathbb{R}}^{4}$, whose Lie algebra is 1-graded: Lie $G=\mathfrak{g}_{-1} \oplus \mathfrak{g}_{0} \oplus \mathfrak{g}_{1}=\mathbb{R}^{4} \oplus \operatorname{LieCO}(1,3) \oplus \mathbb{R}^{4 *}$, where $\mathbb{R}^{4 *}$ is the dual of $\mathbb{R}^{4}$ and $C O(1,3)=$ $S O(1,3) \times \mathbb{R}^{+} /\{0\}$. The structure group $H$ of $\mathcal{P}$ is the (parabolic) subgroup of $G$ stabilising the points of $\overline{\mathbb{R}}^{n}$, which is then the homogeneous space $\sim G / H$. Its Lie algebra is $\operatorname{LieH}=\operatorname{LieCO}(1,3) \oplus \mathbb{R}^{4 *}$. The Cartan connection and its curvature split along the grading.

We will not enter into more details, ${ }^{19}$ save to say that one can write a $\mathcal{H}$-invariant YM-like Lagrangian for gauge conformal gravity, which reduces to the well-known Lagrangian of Weyl gravity when $\bar{A}$ is the normal conformal Cartan connection:

$$
L_{\text {Conf }}(\bar{A})=\frac{1}{2} \operatorname{Tr}(\bar{F} \wedge * \bar{F}) \quad \underset{\text { normal }}{\stackrel{\bar{A}=\bar{A}(e)}{\Rightarrow}} \quad L_{\text {Weyl }}(e)=\frac{1}{2} \operatorname{Tr}(W \wedge * W),
$$

where $W$ is the Weyl tensor. Therefore, the YM equation $D^{\bar{A}} * \bar{F}=0$ for the normal Cartan connection $\bar{A}(e)$ is exactly equivalent to the Bach equation of Weyl gravity. Here again, the ground state of the theory is the conformally (and Cartan) flat homogeneous space $\overline{\mathbb{R}}^{4}$.

The spinors naturally associated to conformal Cartan geometry are $S U(2,2)$ spinors that are none other than twistors. The spin representation of $\bar{A}(e)$ is the twistor connection 1-form, and the associated covariant derivative is the twistor connection (or twistor transport). See e.g. [56].

\footnotetext{
${ }^{19}$ For which we refer to chapter 7 of [33]. For a shorter presentation see section 5 of [55] and references therein.
} 
After this brief overview of the geometry underlying gauge theories, we should remark that it is often necessary to reduce or restrict their gauge symmetries. Indeed, despite their powerful heuristic utility, gauge symmetries are not directly observable: the physical d.o.f. and quantities are gauge-invariants. To identify these may be a highly non-trivial task, so that gauge symmetry reduction methods are an important technical staple of gauge field theory. In our last section, we review one such method.

\section{The dressing field method}

The dressing field method (DFM) is a tool of gauge symmetry reduction quite distinct from other means to achieve similar results, such as gauge fixing or SSB mechanisms. First formulated in [57] and [58], a short review is [55]. We here report the main results of the DFM, in the hope that it becomes more widely known.

\subsection{Reduction of gauge symmetries}

We begin by defining the central object of the DFM. We consider a a $\mathcal{H}$-gauge theory based on a bundle $\mathcal{P}(\mathcal{M}, H)$.

Definition 10. Suppose $\exists$ subgroups $K \subseteq H$ of the structure group, to which corresponds a subgroup $\mathcal{K} \subset \mathcal{H}$ of the gauge group, and $G$ s.t. $K \subseteq G \subseteq H$. A dressing field is a map $u: \mathcal{P} \rightarrow G$ defined by its $K$-equivariance $R_{k}^{*} u=k^{-1} u$. Denote the space of $G$-valued $K$-dressing fields on $\mathcal{P}$ by $\operatorname{Dr}[G, K]$. It follows immediately that the $\mathcal{K}$-gauge transformation of a dressing field is $u^{\gamma}=\gamma^{-1} u$, for $\gamma \in \mathcal{K}$.

Given the existence of a dressing field, we have the following

Proposition 11. From $A \in \mathcal{A}$ and $\alpha \in \Omega_{\text {tens }}^{\bullet}(\mathcal{P}, V)$, one defines the dressed fields

$$
A^{u}:=u^{-1} A u+u^{-1} d u, \quad \text { and } \quad \alpha^{u}:=\rho(u)^{-1} \alpha,
$$

which have trivial $K$-equivariance and are $K$-horizontal, thus are $K$-basic on $\mathcal{P}$. It follows that they are $\mathcal{K}$-invariant: $\left(A^{u}\right)^{\gamma}=A^{u}$ and $\left(\alpha^{u}\right)^{\gamma}=\alpha^{u}$, for $\gamma \in \mathcal{K}$, as is easily checked. The dressed curvature $F^{u}=d A^{u}+{ }^{1} / 2\left[A^{u}, A^{u}\right]=u^{-1} F u$ appears when squaring the dressed covariant derivative $D^{A^{u}}:=d+\rho_{*}\left(A^{u}\right)=\rho(u)^{-1} D^{A}$, and satisfies the Bianchi identity $D^{A^{u}} F^{u}=0$.

In case the equivariance group of $u$ is $K=H, \alpha^{u} \in \Omega_{\text {basic }}^{\bullet}(\mathcal{P}, V)$ and $A^{u} \in \Omega_{\text {basic }}^{1}(\mathcal{P}, \mathrm{Lie} H)$ are $\mathcal{H}$-invariant, thus project as forms on $\mathcal{M}$. The above results make sense for $G \supset H$ if we assume that representations $(V, \rho)$ of $H$ extend to representations of $G$.

Let us emphasize an important fact: It should be clear from its definition that $u \notin \mathcal{K}$, so that (26) are not gauge transformations, despite the formal resemblance. This means, in particular, that the dressed connection is no more a $H$-connection, $A^{u} \notin \mathcal{A}$, and a fortiori is not a point in the gauge $\mathcal{K}$-orbit $O_{\mathcal{K}}[A]$ of $A$, so that $A^{u}$ must not be confused with a gauge-fixing of $A$. 
Let us indulge in a brief digression that is also a segue to the results of the next section. In the BRST framework, infinitesimal gauge transformations are encoded as $s A=-D^{A} \boldsymbol{v}$ and $\boldsymbol{s} \alpha=-\rho_{*}(\boldsymbol{v}) \alpha$, where $\boldsymbol{s}$ is the nilpotent BRST operator and $\boldsymbol{v}$ the ghost field. The latter has values in Lie $\mathcal{H}$ and satisfies $s \boldsymbol{v}+1 / 2[\boldsymbol{v}, \boldsymbol{v}]=0$. For this reason, $\boldsymbol{s}$ is best interpreted geometrically as the de Rham derivative on $\mathcal{H}$ and $\boldsymbol{v}$ as its Maurer-Cartan form [59]. One shows that, at a purely formal level, the dressed variables satisfy a modified BRST ${ }^{u}$ algebra: $s A^{u}=-D^{A^{u}} \boldsymbol{v}^{u}$ and $\boldsymbol{s} \alpha^{u}=-\rho_{*}\left(\boldsymbol{v}^{u}\right) \alpha^{u}$, where one defines the dressed ghost $\boldsymbol{v}^{u}:=u^{-1} \boldsymbol{v} u+u^{-1} \boldsymbol{s} u$.

In the special where case $u$ is a $H$-dressing, its defining equivariance translates as $s u=-v u$. Then the dressed ghost is $v^{u}=0$ and BRST ${ }^{u}$ is trivial, $\boldsymbol{s} A^{u}=0$ and $\boldsymbol{s} \alpha^{u}=0$. In the more general case of a $K$-dressing $u$ achieving only partial gauge reduction, $\mathrm{BRST}^{u}$ only makes sense if it encodes residual gauge transformations of the dressed fields (26). We may then inquire on when the latter are well defined.

\subsection{Residual gauge transformations}

To speak meaningfully about residual gauge transformations of the dressed fields, we need some assumptions. First, we must asssume that $K$ is a normal subgroup, $K \triangleleft H$, so that the $J:=H / K$ is indeed a group, to which corresponds the (residual) gauge subgroup $\mathcal{J} \subset \mathcal{K}$.

Now, the action of $\mathcal{J}$ on the initial variables $A$ and $\alpha$ is known. Therefore what will determine the $\mathcal{J}$-residual gauge transformations of the dressed fields is the action of $\mathcal{J}$ on the dressing field. And this in turn is determined by its $J$-equivariance. In that regard consider the following

Proposition 12. Suppose the dressing field $u$ has $J$-equivariance given by $R_{j}^{*} u=j^{-1} u j$. Then the dressing field has $\mathcal{J}$-gauge transformation $u^{\eta}=\eta^{-1} u \eta$ for $\eta \in \mathcal{J}$, and the residual gauge transformations of the dressed fields are: $\left(A^{u}\right)^{\eta}=\eta^{-1} A^{u} \eta+\eta^{-1} d \eta$ and $\left(\alpha^{u}\right)^{\eta}=\rho(\eta)^{-1} \alpha^{u}$, so in particular $\left(F^{u}\right)^{\eta}=\eta^{-1} F^{u} \eta$.

In the BRST language, the normality of $K$ in $H$ implies $v=v_{K}+v_{J}$, where $v_{K}$ and $v_{J}$ are respectively Lie $\mathcal{K}$ and Lie $\mathcal{J}$ valued, and in accordance $s=s_{K}+s_{J}$. The defining $K$-equivariance of the dressing field translates as $s_{K} u=-v_{K} u$, while its $J$-equivariance assumed in Proposition 12 is encoded as $\boldsymbol{s}_{J} u=\left[u, \boldsymbol{v}_{J}\right]$. The dressed ghost field is thus $\boldsymbol{v}^{u}=u^{-1}\left(\boldsymbol{v}_{K}+\boldsymbol{v}_{J}\right) u+u^{-1}\left(\boldsymbol{s}_{K}+\boldsymbol{s}_{J}\right) u=$ $u^{-1}\left(\boldsymbol{v}_{K}+\boldsymbol{v}_{J}\right) u+u^{-1}\left(-\boldsymbol{v}_{K} u+\left[u, v_{J}\right]\right)=\boldsymbol{v}_{J}$. Therefore, the modified (actually reduced) BRST ${ }^{u}$ algebra is: $\boldsymbol{s}_{J} A^{u}=-D^{A^{u}} \boldsymbol{v}_{J}$ and $\boldsymbol{s}_{J} \alpha^{u}=-\rho_{*}\left(\boldsymbol{v}_{J}\right) \alpha^{u}$. As expected, it encodes the residual gauge transformations of the dressed fields.

Consider the Lagrangian $L(A, \varphi)$ of our initial $\mathcal{H}$-gauge theory. Due to the $\mathcal{H}$-invariance of the Lagrangian, which holds as a formal property of $L$ as a functional, and due to the formal similarity between a gauge transformation and a dressing operation, we have that $L(A, \varphi)=L\left(A^{u}, \varphi^{u}\right)$. That is, the $\mathcal{H}$-gauge theory can be rewritten in terms of $\mathcal{K}$-invariant variables, which means that it becomes a $\mathcal{J}$-gauge theory: the $\mathcal{K}$-gauge symmetry sector has been neutralised. This is how, very simply, the DFM achieves gauge symmetry reduction without gauge fixing or SSB. 
We end this section with a short historical retrospective. The DFM provides a framework for an idea that has resurfaced several times. The earliest example of (abelian) dressing field is the so-called Stueckelberg field, introduced in [60, 61], see [62] for a review. Dirac's gauge-invariant formulation of QED [63] - see also [64] (section 80) - is another (abelian) application of the DFM. It also appeared in QCD, e.g. in the so-called "proton spin decomposition controversy" [65-67], or in the issue of the construction of physical quark states [68].

Most notably, it featured repeatedly in reformulations of theories undergoing SSB [69-75]. In recent years, the fact that such reformulations cast a new light on these theories, on the electroweak model in particular, has been appreciated by philosophers of physics [76-80]. The most recent unwitting application of the DFM are the so-called "edge modes" invoked as a way to deal with the problem of boundaries in the study of the symplectic structure of gauge theories [52, 81-84]

\section{Conclusion}

After having dwelled mainly on the elementary technical aspects of the differential geometry of classical gauge field theory, let us conclude with remarks of a more philosophical nature.

As is well-known, the heuristic that led Einstein to GR is the principle of general covariance (of the field equations), i.e. invariance of the theory (the Lagrangian) under pointwise coordinate changes, a.k.a passive diffeomorphisms. A similar heuristic at the heart of Yang-Mills-Utiyama gauge theories is the gauge principle, according to which the theory is required to be invariant under local transformations of the field variables, which are called gauge symmetries. From a geometric perspective (section 4.1), this may be first understood as requiring invariance of the Lagrangian of the theory under gluings across $\mathcal{M}$ of local representatives of global objects living on $\mathcal{P}$. We may call this "invariance under passive gauge transformations", as it is conceptually analogous to passive diffeomorphisms: in both cases it is a matter of different "observers" having situated access to, and thus different viewpoints on, globally defined geometric objects of $\mathcal{M}$ or $\mathcal{P} .{ }^{20}$ The general covariance principle (GCP) and the passive gauge principle (PGP) are then principles of "democratic epistemic access" to objective geometric structures, and to the physics their dynamic encodes.

But the story doesn't end there. In GR, passive diffeomorphisms/coordinate changes are undistinguishable from active diffeomorphisms, so that the passive invariance of the theory imply its active invariance. ${ }^{21}$ But the latter has a much deeper physical interpretation, to wit: that it is not the manifold $\mathcal{M}$ itself that encodes physics, but its whole $\operatorname{Diff}(\mathcal{M})$-class. As we have briefly alluded to in section 3.2, this is the key insight at the heart of Einstein's "Hole argument" - a pivotal moment in the history of the elaboration of GR - which implies that points of $\mathcal{M}$ are unphysical, only structural relations between points are. By extension, field configurations on $\mathcal{M}$ are unphysical, only the relative configurations of fields over $\mathcal{M}$ are. The choice of a manifold $\mathcal{M}$ is then itself a "coordinatisation" of sort, i.e. a conventional representational choice from which relativistic physics is ultimately independent. This is often phrased as GR being background independent.

\footnotetext{
${ }^{20} \mathrm{~A}$ choice of local section $\sigma: U \subset \mathcal{M} \rightarrow \mathcal{P}$, with which one pulls-back global objects on $\mathcal{P}$ as local representatives, is a 'choice of gauge' and is equivalent to a choice of local bundle coordinate chart. It is thus exactly analogous to a choice of local coordinates on $U$ with which one represents intrinsic geometric objects on $\mathcal{M}$.

${ }^{21}$ Actually, invariance under volume preserving diffeomorphisms of $\mathcal{M}$.
} 
A very similar analysis holds for gauge theories. If points of $\mathcal{M}$ are structureless, one can see $\mathcal{P}$ as an "enriched" spacetime whose points have an internal structure (the fiber). As we have seen, passive gauge transformations (gluings) are formally indistinguishable from active gauge transformations by vertical automorphisms $\operatorname{Aut}_{v}(\mathcal{P})$. So, the passive invariance of the theory implies its active invariance. The latter has the stronger interpretive implication that it is not the bundle $\mathcal{P}$ that encodes the enriched physical structure of spacetime, but its $\operatorname{Aut}_{v}(\mathcal{P})$-class. Meaning that individual points in the fibers of $\mathcal{P}$ are unphysical, only the structural relations between points of the fibers are. By extension field configurations along fibers of $\mathcal{P}$ are unphysical, only relative configurations of fields along fibers are. A choice of $\mathcal{P}$ may be seen as a representational convention from which gauge physics is in fine independent: we have a form of abstract background independence.

It then appears that reasonable principles of democratic epistemic access (GCP and PGP) morph into, or imply, a strong ontological proposition: A principle of physical dynamical relationalism, which can be phrased as requiring a strong background independence, i.e. independence of physics from any non-dynamical geometric structures.

We may remark that the group of spacetime diffeomorphisms $\operatorname{Diff}(\mathcal{M})$ and the gauge group $\mathcal{H} \simeq \operatorname{Aut}_{v}(\mathcal{P})$ are terms of the SES (15), and are "unified" within the group $\operatorname{Aut}(\mathcal{P}) \subset \operatorname{Diff}(\mathcal{P})$ of bundle automorphisms. It would be tempting to think that general-relativisitic-gauge theories must satisfy a principle of $\operatorname{Aut}(\mathcal{P})$-invariance. But surprisingly, at least in the case of theories without matter fields, this seems to be too strong a constraint: indeed, $\operatorname{Aut}(\mathcal{P})$-invariant Lagrangian $L(A)$ are variationaly trivial, i.e. their field equations are identically satisfied, and they encode only topological informations of $\mathcal{P}$ and $\mathcal{M}$ [85]. This would make a $\operatorname{Aut}(\mathcal{P})$-invariance principle viable only for topological gauge field theory. It would be interesting to see if such a principle is viable for variationaly non-trivial coupled theories $L(A, \varphi, \psi)$.

All this being said, not all symmetries have the same status. It is well-known in philosophy of relativistic physics that the so-called "Kretschmann objection" against the GCP, according to which any theory could in principle be rewritten so as to be generally covariant, led to the recognition that one should distinguish artificial general covariance (which is forced onto a theory) from substantive general covariance (which is integral to a theory) [38]. The next point is to identify and implement demarcation criteria. Each such criterion would capture a key aspect of the physical meaning of substantive general covariance (such as the ones discussed above).

In the past 15 to 20 years it has been clear that a "generalised Kretschmann objection" can be leveraged against the PGP: Physicist know of several ways to implement a gauge symmetry in a theory that has none to begin with (e.g. the Stueckelberg trick [62]), then how could gauge symmetries have any deep physical meaning? Again, one is led to distinguish artificial from substantive gauge symmetries [86, 87], and left with the task of identifying one or several demarcation criteria that would capture the physical content of substantive gauge symmetries, and hopefully are easily implementable. One such criterion seem to make broad consensus: the trade-off gauge-invariance $v s$ locality. It appears indeed that one can get rid of an artificial gauge symmetry without losing the locality of the theory, while it is not so for substantive gauge symmetries. In substantive gauge theories, physical d.o.f. are encoded non-locally [88-90] (a phenomenon a priori distinct from quantum non-locality). The Aharonov-Bohm effect is often cited as a prototypical circumstance where this is made clear. The DFM happens to provide a systematic way to implement this criterion. For a broader discussion of this issue see [91] and references therein. 
We have laid here only the basics of the differential geometry of gauge theories. Differential geometric methods have proved powerful, even indispensable, in the study of non-perturbative effects in quantum gauge field theory. For example, the so-called gauge anomalies, initially computed via Feynman diagrams, are now understood in terms of the cohomology of the gauge group (which is what BRST cohomology is) [59, 92]. Many landmark results testify to the fruitful interplay between differential geometry and theoretical physics: gauge-fixing and Gribov ambiguity [93, 94], anomalies and Atiyah-Singer index theorem [92], Donaldson's theory of smooth 4D manifolds and YM instantons, cobordism and topological quantum field theory, etc... Algebraic, topological and differential geometric concepts have come to still greater prominence in the era of string theory. Given the historical fact of the cross-fertilisation of mathematics and physics, it seems unavoidable that higher mathematics are bound to play an ever increasing role in theoretical physics.

\section{Acknowledgments}

The author thanks the organisers of the XVI Modave Summer School in Mathematical Physics for their generous invitation to give the lectures from which these notes are inspired.

\section{References}

[1] E. Cartan, Les récentes généralisations de la notion d'espace, Bull. Sci. Math. 48 (1924) $825-861$.

[2] H. Whitney, Sphere-spaces, Proceedings of the National Academy of Sciences 21 (1935), no. 7 464-468, [https: //www . pnas . org/content/21/7/464 . full.pdf].

[3] H. Whitney, On the theory of sphere-bundles, Proceedings of the National Academy of Sciences of the United States of America 26 (02, 1940) 148-153.

[4] C. Ehresmann, Sur la théorie des espaces fibrés, Colloque de topologie algébrique du CNRS, Paris (1947) 3-15.

[5] N. STEENROD, The Topology of Fibre Bundles. (PMS-14). Princeton University Press, 1951.

[6] C. Ehresmann, Les connexions infinitésimales dans un espace fibré différentiable, Colloque de topologie de Bruxelles (1950) 29-55.

[7] S. Kobayashi, Theory of connections, Annali di Matematica Pura ed Applicata 43 (Dec., 1957) 119-194.

[8] S. Kobayashi and K. Nomizu, Foundations of Differential Geometry, vol. I. Wiley \& Sons, 1963.

[9] S. Kobayashi and K. Nomizu, Foundations of Differential Geometry, vol. II. Wiley \& Sons, 1969. 
[10] H. Weyl, Gravitation and electricity, Sitzungsber. Preuss. Akad. Wiss. Berlin (Math. Phys.) 1918 (1918) 465.

[11] H. Weyl, A New Extension of Relativity Theory, Annalen Phys. 59 (1919) 101-133. [Annalen Phys.364,101(1919)].

[12] H. Weyl, Gravitation and the electron, Proceedings of the National Academy of Sciences $\mathbf{1 5}$ (1929), no. 4 323-334, [https: //www . pnas . org/content/15/4/323. full.pdf].

[13] C. N. Yang and R. L. Mills, Conservation of isotopic spin and isotopic gauge invariance, Phys. Rev. 96 (Oct, 1954) 191-195.

[14] R. Shaw. PhD thesis, University of Cambridge, September, 1955.

[15] R. Utiyama, Invariant Theoretical Interpretation of Interaction, Phys. Rev. 101 (Mar, 1956) 1597-1607.

[16] H. Yukawa, On the Interaction of Elementary Particles I, Proc. Phys. Math. Soc. Jap. 17 (1935) 48-57.

[17] S. Weinberg, A model of leptons, Phys. Rev. Lett. 19 (Nov, 1967) 1264-1266.

[18] H. Fritzsch, M. Gell-Mann, and H. Leutwyler, Advantages of the color octet gluon picture, Physics Letters B 47 (1973), no. 4365 - 368.

[19] R. Brout and F. Englert, Broken symmetry and the mass of the gauge vector mesons, Physical Review Letters 13 (1964) 321-323.

[20] P. W. Higgs, Broken symmetry and the mass of gauge bosons, Physical Review Letters 13 (1964) 508-509.

[21] G. S. Guralnik, C. R. Hagen, and T. W. B. kibble, Global conservation laws and massless particles, Physical Review Letters 13 (1964) 585-587.

[22] D. Gross and F. Wilczek, Ultraviolet behavior of non-abelian gauge theories, Phys. Rev. Lett. 30 (Jun, 1973) 1343-1346.

[23] H. D. Politzer, Reliable perturbative results for strong interactions?, Phys. Rev. Lett. 30 (Jun, 1973) 1346-1349.

[24] L. O'Raifeartaigh, The Dawning of Gauge Theory. Princeton Series in Physics. Princeton University Press, 1997.

[25] A. Pais, Inward Bound: Of Matter And Forces In The Physical World. UK, Clarendon Press. USA, Oxford University Press, 1986.

[26] P. Pesic, Beyond Geometry: Classic Papers from Riemann to Einstein. Dover Publication, Inc., 2007. 
[27] C. M. Marle, The works of Charles Ehresmann on connections: from Cartan connections to connections on fibre bundles, in Geometry and Topology of Manifolds, vol. 76. Banach Center Publication, 2007.

[28] T. T. Wu and C. N. Yang, Concept of Nonintegrable Phase Factors and Global Formulation of Gauge Fields, Phys. Rev. D 12 (1975) 3845-3857.

[29] A. Trautman, Fiber Bundles, Gauge Field and Gravitation, in General Relativity and Gravitation, vol. 1. Plenum Press, New-York, 1979.

[30] T. Eguchi, P. Gilkey, and A. Hanson, Gravitation, gauge theories and differential geometry, Physics Reports 66 (1980), no. 6213 - 393.

[31] M. Göckeler and T. Schücker, Differential Geometry, Gauge Theory and Gravity. Cambridge Monographs on Mathematical Physics. Cambridge University Press, 1987.

[32] M. Nakahara, Geometry, Topology and Physics. 2nd Edition. Graduate Student Series in Physics. Taylor \& Francis, 2003.

[33] R. W. Sharpe, Differential Geometry: Cartan's Generalization of Klein's Erlangen Program, vol. 166 of Graduate text in Mathematics. Springer, 1996.

[34] A. Cap and J. Slovak, Parabolic Geometries I: Background and General Theory, vol. 1 of Mathematical Surveys and Monographs. American Mathematical Society, 2009.

[35] D. K. Wise, Symmetric space, cartan connections and gravity in three and four dimensions, SIGMA 5 (2009) 080-098.

[36] D. K. Wise, MacDowell-Mansouri gravity and Cartan geometry, Classical and Quantum Gravity 27 (2010) 155010.

[37] S. Lazzarini and T. Masson, Connections on Lie algebroids and on derivation-based noncommutative geometry, J. Geom. Phys 62 (2012) 387.

[38] O. Pooley, Towards a Theory of Spacetime Theories. Einstein Studies, vol 13., ch. Background Independence, Diffeomorphism Invariance and the Meaning of Coordinates., pp. 105-143. Birkhauser, New York, NY, 2017.

[39] H. Lawson and M. Michelsohn, Spin geometry. 1998.

[40] D. Lehmkuhl, Why Einstein did not believe that general relativity geometrizes gravity, Studies in History and Philosophy of Science Part B: Studies in History and Philosophy of Modern Physics 46 (2014) 316 - 326.

[41] E. Cartan and A. Einstein, Élie Cartan - Albert Einstein: letters on absolute parallelism, 1929-1932. Princeton University Press, 1979.

[42] C. A. Martin, Gauge principles, gauge arguments and the logic of nature, Proceedings of the Philosophy of Science Association 3 (2002) 221-234. 
[43] R. Penrose and W. Rindler, Spinors and Space-Time, vol. 1. Cambridge University Press, 1984.

[44] R. Penrose and W. Rindler, Spinors and Space-Time, vol. 2. Cambridge University Press, 1986.

[45] E. Witten, Interacting field theory of open superstrings, Nuclear Physics B 276 (1986), no. 2 $291-324$.

[46] G. J. Zuckerman, Action principles and global geometry, Conf. Proc. C 8607214 (1986) 259-284.

[47] C. Crnkovic and E. Witten, Three hundred years of gravitation, ch. Covariant description of canonical formalism in geometrical theories, pp. 676-684. Cambridge University Press, 1987.

[48] C. Crnkovic, Symplectic Geometry of the Covariant Phase Space, Superstrings and Superspace, Class. Quant. Grav. 5 (1988) 1557-1575.

[49] E. De Paoli and S. Speziale, A gauge-invariant symplectic potential for tetrad general relativity, Journal of High Energy Physics 2018 (2018), no. 740.

[50] R. Oliveri and S. Speziale, A note on dual gravitational charges, https://arxiv.org/abs/2010.01111 (2020).

[51] L. Freidel, M. Geiller, and D. Pranzetti, Edge modes of gravity III: Corner simplicity constraints, arXiv:2007.12635.

[52] W. Donnelly and L. Freidel, Local subsystems in gauge theory and gravity, Journal of High Energy Physics 2016 (2016), no. 9102.

[53] R. Oliveri and S. Speziale, Boundary effects in general relativity with tetrad variables, General Relativity and Gravitation 52 (2020), no. 883.

[54] J. François, Bundle geometry of the connection space, covariant Hamiltonian formalism, the problem of boundaries in gauge theories, and the dressing field method, https://arxiv.org/abs/2010.01597 (2020) [arXiv: 2010 . 01597].

[55] J. Attard, J. François, S. Lazzarini, and T. Masson, Foundations of Mathematics and Physics one Century After Hilbert : New Perspectives, ch. The dressing field method of gauge symmetry reduction, a review with examples. Springer, 2018.

[56] J. Attard and J. François, Tractors and Twistors from conformal Cartan geometry: a gauge theoretic approach II. Twistors, Class. Quantum Grav. 34 (March, 2017) [arXiv: 1611.03891].

[57] C. Fournel, J. François, S. Lazzarini, and T. Masson, Gauge invariant composite fields out of connections, with examples, Int. J. Geom. Methods Mod. Phys. 11 (2014), no. 11450016. 
[58] J. François, Reduction of gauge symmetries: a new geometrical approach. Thesis, Aix-Marseille Université, September, 2014.

[59] L. Bonora and P. Cotta-Ramusino, Some remark on BRS transformations, anomalies and the cohomology of the Lie algebra of the group of gauge transformations, Commun. Math. Phys. 87 (1983) 589-603.

[60] E. Stueckelberg, Interaction energy in electrodynamics and in the field theory of nuclear forces (Part I), Helv. Phys. Acta 11 (1938) 225-244.

[61] E. Stueckelberg, Interaction forces in electrodynamics and in the field theory of nuclear forces (Part II-III), Helv. Phys. Acta 11 (1938) 299-328.

[62] R. Ruegg and M. Ruiz-Altaba, The stueckelberg field, Int. J. Mod. Phys A 19 (2004) 3265-3347.

[63] P. A. M. Dirac, Gauge-invariant formulation of quantum electrodynamics, Canadian Journal of Physics 33 (1955) 650-660.

[64] P. A. M. Dirac, The principles of Quantum Mechanics. Oxford University Press, 4th edn ed., 1958.

[65] C. Lorcé, Geometrical approach to the proton spin decomposition, Physical Review D 87 (2013) 034031.

[66] E. Leader and C. Lorcé, The angular momentum controversy: What is it all about and does it matter?, Physics Reports 514 (2014) 163-248.

[67] J. François, S. Lazzarini, and T. Masson, Nucleon spin decomposition and differential geometry, Phys. Rev. D 91 (Feb, 2015) 045014.

[68] M. Lavelle and D. McMullan, Constituent quarks from QCD, Physics Reports 279 (1997) $1-65$.

[69] P. W. Higgs, Spontaneous symmetry breakdown without massless bosons, Phys. Rev. 145 (May, 1966) 1156-1163.

[70] T. W. B. Kibble, Symmetry breaking in nonAbelian gauge theories, Phys. Rev. 155 (1967) 1554-1561.

[71] J. Frohlich, G. Morchio, and F. Strocchi, Higgs phenomenon without symmetry breaking order parameter, Nuclear Physics B 190 (1981), no. 3553 - 582.

[72] M. Lavelle and D. McMullan, Observables and gauge fixing in spontaneously broken gauge theories, Physics Letters B 347 (1995), no. 189 - 94.

[73] L. D. Faddeev, An Alternative Interpretation of the Weinberg-Salam Model, in Progress in HighEnergy Physics and Nuclear Safety (V. Begun, L. L. Jenkovszky, and A. Polański, eds.), (Dordrecht), pp. 3-8, Springer Netherlands, 2009. 
[74] A. Ilderton, M. Lavelle, and D. McMullan, Symmetry breaking, conformal geometry and gauge invariance, Journal of Physics A: Mathematical and Theoretical 43 (2010), no. 31 312002 .

[75] A. Maas, Brout-englert-higgs physics: From foundations to phenomenology, Progress in Particle and Nuclear Physics 106 (2019) 132 - 209.

[76] C. Smeenk, The Elusive Higgs Mechanism, Philosophy of Science 73 (2006), no. 5 487-499.

[77] H. Lyre, Does the Higgs Mechanism Exists?, International Studies in the Philosophy of Science 22 (2008), no. 2 119-133.

[78] W. Struyve, Gauge invariant accounts of the higgs mechanism, Studies in History and Philosophy of Science Part B: Studies in History and Philosophy of Modern Physics $\mathbf{4 2}$ (2011), no. $4226-236$.

[79] S. Friederich, Gauge symmetry breaking in gauge theories - in search of clarification, European Journal for Philosophy of Science 3 (2013), no. 2 157-182.

[80] S. Friederich, A Philosophical Look at the Higgs Mechanism, J. Gen. Philos. Sci 45 (July, 2014) 335-350.

[81] M. Geiller, Edge modes and corner ambiguities in 3d chern-simons theory and gravity, Nuclear Physics B 924 (2017) 312 - 365.

[82] A. Speranza, Local phase space and edge modes for diffeomorphism-invariant theories, Journal of High Energy Physics 2018 (2018), no. 221.

[83] P. Mathieu, L. Murray, A. Schenkel, and N. J. Teh, Homological perspective on edge modes in linear yang-mills and chern-simons theory, Letters in Mathematical Physics 110 (2020), no. 7 1559-1584.

[84] S. Murgueitio Ramírez and N. Teh, Abandoning Galileo's Ship: The quest for non-relational empirical significance, The British Journal for the Philosophy of Science (June, 2020).

[85] M. Castrillón López, J. Muñoz Masqué, and T. Ratiu, Gauge invariance and variational trivial problems on the bundle of connections, Differential Geometry and its Applications 19 (2003), no. 2127 - 145 .

[86] J. B. Pitts, General Covariance, Artificial Gauge Freedom and Empirical Equivalence. $\mathrm{PhD}$ thesis, Graduate School of the University of Notre Dame, 2008.

[87] J. B. Pitts, Empirical equivalence, artificial gauge freedom and a generalized kretschmann objection, .

[88] R. Healey, Gauging What's Real: The Conceptual Foundation of Contemporary Gauge Theories. Oxford University Press, 2009. 
[89] J. Nguyen, N. J. Teh, and L. Wells, Why surplus structure is not superfluous, The British Journal for the Philosophy of Science 71 (June, 2020) 665-695.

[90] J. Dougherty, Sameness and separability in gauge theories, Philosophy of Science 84 (December, 2017) 1189-1201.

[91] J. François, Artificial versus Substantial Gauge Symmetries: A Criterion and an Application to the Electroweak Model, Philosophy of Science 86 (2019), no. 3 472-496, [https://doi.org/10.1086/703571].

[92] R. A. Bertlmann, Anomalies In Quantum Field Theory, vol. 91 of International Series of Monographs on Physics. Oxford University Press, 1996.

[93] V. N. Gribov, Quantization of non-abelian gauge theories, Nucl. Phys. B 139 (1978) 1-19.

[94] I. M. Singer, Some remark on the gribov ambiguity, Commun. Math. Phys. 60 (1978) 7-12. 